\title{
Signatures of the non-Maxwellian $\kappa$-distributions in optically thin line spectra ${ }^{\star}$
}

\section{Theory and synthetic Fe IX-XIII spectra}

\author{
J. Dudík ${ }^{1,2, \star \star}$, G. Del Zanna ${ }^{1}$, H. E. Mason ${ }^{1}$, and E. Dzif̌ćáková ${ }^{3}$ \\ ${ }^{1}$ Department of Applied Mathematics and Theoretical Physics, CMS, University of Cambridge, Wilberforce Road, \\ Cambridge CB3 0WA, UK \\ e-mail: j.dudik@damtp.cam.ac.uk \\ 2 Department of Astronomy, Physics of the Earth and Meteorology, Faculty of Mathematics, Physics and Informatics, \\ Comenius University, 84248 Bratislava, Slovak Republic \\ 3 Astronomical Institute, Academy of Sciences of the Czech Republic, 25165 Ondřejov, Czech Republic \\ e-mail: elena@asu.cas.cz
}

Received 3 May 2014 / Accepted 24 July 2014

\begin{abstract}
Aims. We investigate the possibility of diagnosing the degree of departure from the Maxwellian distribution using single-ion spectra originating in astrophysical plasmas in collisional ionization equilibrium.

Methods. New atomic data for excitation of Fe IX - Fe XIII are integrated under the assumption of a $\kappa$-distribution of electron energies. Diagnostic methods using lines of a single ion formed at any wavelength are explored. Such methods minimize uncertainties from the ionization and recombination rates, as well as the possible presence of non-equilibrium ionization. Approximations to the collision strengths are also investigated.

Results. The calculated intensities of most of the Fe IX - Fe XIII EUV lines show consistent behaviour with $\kappa$ at constant temperature. Intensities of these lines decrease with $\kappa$, with the vast majority of ratios of strong lines showing little or no sensitivity to $\kappa$. Several of the line ratios, especially involving temperature-sensitive lines, show a sensitivity to $\kappa$ that is of the order of several tens of per cent, or, in the case of Fe IX, up to a factor of two. Forbidden lines in the near-ultraviolet, visible, or infrared parts of the spectrum are an exception, with smaller intensity changes or even a reverse behaviour with $\kappa$. The most conspicuous example is the Fe X $6378.26 \AA$ red line, whose intensity incerases with $\kappa$. This line is a potentially strong indicator of departures from the Maxwellian distribution. We find that it is possible to perform density diagnostics independently of $\kappa$, with many Fe XI, Fe XII, and Fe XIII line ratios showing strong density-sensitivity and negligible sensitivity to $\kappa$ and temperature. We also tested different averaging of the collision strengths. It is found that averaging over 0.01 interval in $\log (E[\mathrm{Ryd}])$ is sufficient to produce accurate distribution-averaged collision strengths $\Upsilon(T, \kappa)$ at temperatures of the ion formation in ionization equilibrium.
\end{abstract}

Key words. Sun: UV radiation - Sun: X-rays, gamma rays - Sun: corona - Sun: transition region radiation mechanisms: non-thermal

\section{Introduction}

The central assumption when analyzing spectra originating from optically thin astrophysical plasmas in collisional ionization equilibrium is that the electron distribution function is Maxwellian and therefore that the equilibrium is always ensured locally. This may, however, not be true if there are correlations between the particles in the system. These correlations may be induced by any long-range interactions in the emitting plasma (Collier 2004; Livadiotis \& McComas 2009, 2010, 2011, 2013), such as wave-particle interactions, shocks, or particle acceleration and associated streaming of fast, weakly collisional particles from the reconnection site. Under such conditions, the distribution function may depart from the Maxwellian one.

Although still debatable (Storey et al. 2014; Storey \& Sochi 2014), a claim has been made that the $\kappa$-distributions,

\footnotetext{
* Appendices are available in electronic form at

http://www . aanda.org

$\star \star$ RS Newton International Fellow.
}

characterized by a high-energy tail, can explain the observed spectra of planetary nebulae (Binette et al. 2012; Nicholls et al. 2012, 2013; Dopita et al. 2013). The deviations from the Maxwellian distribution required to explain the observed spectra of ions such as O III or S III spectra were small, with $\kappa \approx 20$. These values are much larger than the $\kappa$ measured in situ in the solar wind, which is observed to be strongly non-Maxwellian, typically with $\kappa>2.5$ (e.g. Collier et al. 1996; Maksimovic et al. 1997a,b; Zouganelis 2008; Livadiotis \& McComas 2010; Le Chat et al. 2011).

Since $\kappa$-distributions are observed in the solar wind, a question arises whether these could originate in the solar corona. Although attempts to detect high-energy particles have been made (Feldman et al. 2007; Hannah et al. 2010), their presence is still unclear. Feldman et al. (2007) used bi-Maxwellian spectral modelling of the line intensities of He-like lines observed by SUMER (Wilhelm et al. 1995). The second Maxwellian was assumed to have a temperature of $10 \mathrm{MK}$. The authors argued that the presence of this high-temperature Maxwellian was 
not neccessary to explain the observed spectra. However, the analysis was limited to Maxwellian distributions and did not include the effects of $\kappa$-distributions on the spectra. Hannah et al. (2010) used the RHESSI instrument (Lin et al. 2002) to observe an off-limb quiet-Sun region and derived strong constraints on the number of particles at energies of several keV. However, the derived limits on the emission measure of the plasma at temperatures of several MK are still somewhat large, and increase with increasing $\kappa$.

On the other hand, Scudder \& Karimabadi (2013) argue that stellar coronae above $1.05 R_{\odot}$ should be strongly nonMaxwellian. Dzifčáková et al. (2011) analyzed the Si III line intensities reported by Pinfield et al. (1999) and concluded that the observed spectra can be explained by $\kappa$-distributions with $\kappa \approx 7$ for the active region, and $\kappa \approx 11-13$ for the quiet Sun and coronal hole. The analysis also works under the assumption of a differential emission measure. If these diagnosed values are correct, it could be expected that the solar corona should have values of $\kappa$ between these and those observed in the solar wind. Analysis of the effect of $\kappa$-distributions on the intensities of lines observed by the Hinode/EIS instrument (Culhane et al. 2007) have been made for Fe lines by Dzifčáková \& Kulinová (2010) and for non-Fe lines by Mackovjak et al. (2013). These authors used the atomic data corresponding to CHIANTI v5.3 (Dere et al. 1997; Landi et al. 2006) and v7 (Landi et al. 2012), with the excitation cross sections for $\kappa$-distributions being recovered using an approximate parametric method (Dzifčáková \& Mason 2008). Although some indications of the presence of $\kappa$-distributions were obtained from the O IV-O V and S X-S XI lines, the authors could not exclude the presence of multithermal effects. Futhermore, the diagnostics had to include lines formed at neighbouring ionization stages, because the wavelength range of the EIS instrument is limited (170 $-211 \AA$ and $246 \AA-292 \AA)$. The observed lines of a single ion have by neccessity similar excitation thresholds, which makes their behaviour with $\kappa$ similar. Including the lines formed in neighbouring ionization stages increases the sensitivity of the diagnostics to $\kappa$, but complicates the analysis in the multithermal case. Moreover, the assumption of ionization equilibrium is a possible additional source of uncertainties. The diagnostics of $\kappa$ performed by these authors was also limited by density effects. Nevertheless, Dzifčáková \& Kulinová (2010) showed that some density-sensitive line ratios can be used for diagnostics of electron density independently of the $\kappa$ value.

A successful diagnostic of $\kappa$-distributions has been performed in flares. Kašparová \& Karlický (2009) showed that some coronal sources observed by RHESSI (Lin et al. 2002) in partially occulted flares can be fitted with a $\kappa$-distribution. Oka et al. (2013) showed that $\kappa$-distributions provide a good fit to the observed high-energy tail, and dispense with the need for a low-energy cut-off. However, the authors showed that a nearMaxwellian component is also present at lower energies in the flare studied.

In recent years, significant progress has been made in the calculation of atomic data for collisional excitation of astrophysically important ions (e.g. Liang et al. 2009, 2010, 2012; O'Dwyer et al. 2012; Del Zanna et al. 2010, 2012a; Del Zanna 2011a,b; Del Zanna et al. 2012b; Del Zanna \& Storey 2012, 2013; Del Zanna et al. 2014). These calculations represent significant improvements to previous atomic data, since they include a large number of levels and the associated cascading and resonances. These cross sections for electron excitation are in several cases significantly different from the previous ones. These atomic data will be implemented in the next version 8 of the CHIANTI database. Significant progress has also been

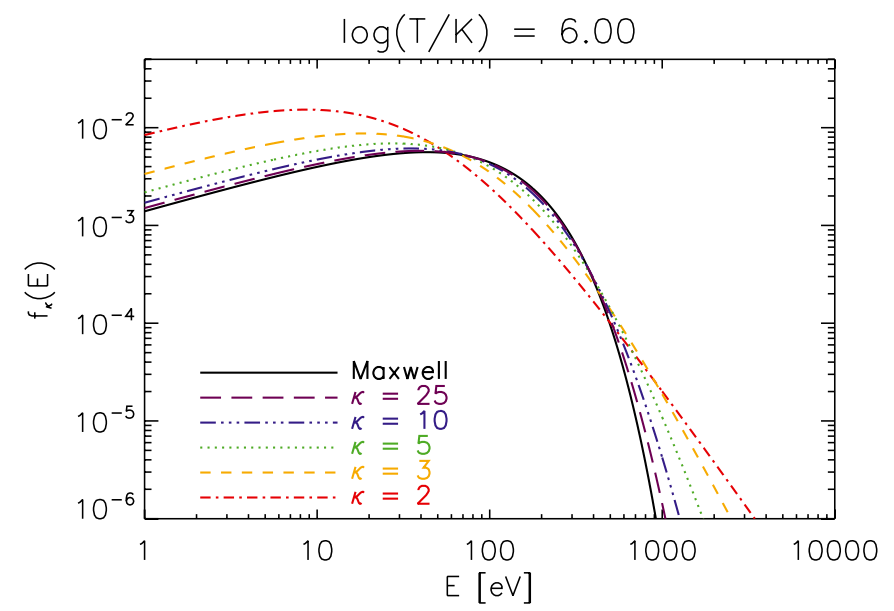

Fig. 1. $\kappa$-distributions with $\kappa=2,3,5,10,25$, and the Maxwellian distribution plotted for $\log (T / \mathrm{K})=6.0$. Different colors and line styles correspond to different values of $\kappa$.

made on line identifications of complex coronal Fe ions (e.g. Del Zanna et al. 2004, 2014; Del Zanna \& Mason 2005; Young 2009; Del Zanna 2010, 2011a, 2012; Del Zanna et al. 2012a).

For these reasons, in this paper we use the original cross sections, which allows us to dispense with the approximative parametric method of Dzifčáková \& Mason (2008). This paper is a first in a series of papers exploring the effect of non-Maxwellian $\kappa$-distributions on the spectra arising from optically thin plasma in collisional ionization equilibrium. Here, we focus on the ions formed in solar and stellar coronae at temperatures of $\approx 1-2 \mathrm{MK}$. The atomic data calculations are exploited by calculating the synthetic spectra for Fe IX - Fe XIII for $\kappa$-distributions throughout the entire wavelength range of spectral line formation. This is done to include the strong forbidden transitions in the visible or infrared part of the electromagnetic spectrum (see e.g. Habbal et al. 2013). The Fe IX-Fe XIII ions are chosen since they produce some of the strongest lines that are observed even during minima of the solar cycle, i.e. in absence of active regions (Landi \& Young 2010).

The paper is structured as follows. The $\kappa$-distributions are described in Sect. 2. The method used to calculate the relative level populations and synthetic spectra using generalized distributionaveraged collision strengths is described in Sect. 3. Averaging of the collision strengths is investigated in Sect. A. Synthetic spectra are presented in Sect. 4, with the influence of $\kappa$-distributions on density-sensitive line ratios studied in Sect. 5. Section 6 explores the possibilities of diagnosing $\kappa$ simultaneously with $T$ using lines of only one ion. Conclusions are given in Sect. 7.

\section{The non-Maxwellian $\kappa$-distributions}

The $\kappa$-distribution (Fig. 1, top) is a distribution of electron energies characterized by a power-law high-energy tail (Owocki \& Scudder 1983; Livadiotis \& McComas 2009, 2013)

$f(E, \kappa) \mathrm{d} E=A_{\kappa} \frac{2}{\sqrt{\pi}\left(k_{\mathrm{B}} T\right)^{3 / 2}} \frac{E^{1 / 2} \mathrm{~d} E}{\left(1+\frac{E}{(\kappa-3 / 2) k_{\mathrm{B}} T}\right)^{\kappa+1}}$,

where $A_{\kappa}=\Gamma(\kappa+1) /\left(\Gamma(\kappa-1 / 2)(\kappa-3 / 2)^{3 / 2}\right)$ is the normalization constant and $k_{\mathrm{B}}=1.38 \times 10^{-16} \mathrm{erg} \mathrm{K}^{-1}$ is the Boltzmann constant; $T$ and $\kappa \in(3 / 2,+\infty)$ are the parameters of the distribution. The parameter $\kappa$ changes the shape of distribution 
function from $\kappa \rightarrow 3 / 2$ corresponding to the highest deviation from Maxwellian distribution, to $\kappa \rightarrow \infty$ corresponding to the Maxwellian distribution.

The mean energy $\langle E\rangle=3 k_{\mathrm{B}} T / 2$ of a $\kappa$-distribution is independent of $\kappa$, so that $T$ can be defined as the temperature also for $\kappa$-distributions. The reader is referred to the paper of Livadiotis \& McComas (2009) for a more detailed discussion of why $T$ can be properly defined as the thermodynamic temperature in the framework of the generalized Tsallis statistical mechanics (Tsallis 1988, 2009).

\section{Method}

\subsection{Spectral line emissivities}

The emissivity $\varepsilon_{j i}$ of an optically thin spectral line arising from a transition $j \rightarrow i, j>i$, in a $k$-times ionized ion of the element $X$, is given by (e.g. Mason \& Monsignori Fossi 1994; Phillips et al. 2008)

$$
\begin{aligned}
\varepsilon_{j i} & =\frac{h c}{\lambda_{j i}} A_{j i} n\left(X_{j}^{+k}\right)=\frac{h c}{\lambda_{j i}} \frac{A_{j i}}{n_{\mathrm{e}}} \frac{n\left(X_{j}^{+k}\right)}{n\left(X^{+k}\right)} \frac{n\left(X^{+k}\right)}{n(X)} A_{X} n_{\mathrm{e}} n_{\mathrm{H}} \\
& =A_{X} G_{X, j i}\left(T, n_{\mathrm{e}}, \kappa\right) n_{\mathrm{e}} n_{\mathrm{H}},
\end{aligned}
$$

where $h \approx 6.62 \times 10^{-27} \operatorname{ergs}$ is the Planck constant, $c \approx$ $3 \times 10^{10} \mathrm{~cm} \mathrm{~s}^{-1}$ represents the speed of light, $\lambda_{j i}$ is the line wavelength, $A_{j i}$ the corresponding Einstein coefficient for the spontaneous emission, $n\left(X_{j}^{+k}\right)$ is the density of the ion $+k$ with electron on the excited upper level $j, n\left(X^{+k}\right)$ the total density of ion $+k$, $n(X) \equiv n_{X}$ the total density of element $X$ whose abundance is $A_{X}$, and $n_{\mathrm{H}}$ the hydrogen density. The function $G_{X, j i}\left(T, n_{\mathrm{e}}, \kappa\right)$ is the contribution function for the line $\lambda_{j i}$. The observed intensity $I_{j i}$ of the spectral line is then given by the integral of emissivity along the line of sight $l$, i.e.

$I_{j i}=\int A_{X} G_{X, j i}\left(T, n_{\mathrm{e}}, \kappa\right) n_{\mathrm{e}} n_{\mathrm{H}} \mathrm{d} l$

where the quantity $E M=\int n_{\mathrm{e}} n_{\mathrm{H}} \mathrm{d} l$ is called the emission measure of the plasma.

\subsection{Ionization rates and ionization equilibrium}

The ratio $n\left(X^{+k}\right) / n_{X}$ is the relative abundance of ion $n\left(X^{+k}\right)$, and is therefore given by the ionization equilibrium. Since the ionization and recombination rates are a function of $T$ and $\kappa$, the ratio $n\left(X^{+k}\right) / n_{X}$ is also a function of $T$ and $\kappa$ (e.g. Dzifčáková 1992, 2002; Wannawichian et al. 2003; Dzif̌ćáková \& Dudík 2013). Here, we use the latest available ionization equilibria for $\kappa$-distributions calculated by Dzifčáková \& Dudík (2013). These use the same atomic data for ionization and recombination as the ionization equilibrium for the Maxwellian distribution available in the CHIANTI database, v7.1 (Landi et al. 2013; Dere 2007; Dere et al. 1997).

Figure 2 shows the behaviour of the relative ion abundances of Fe IX - Fe XIII with $\kappa$. The ionization peaks are in general wider for lower $\kappa$, and are shifted to higher $T$ for low $\kappa=2-3$ (Dzifčáková \& Dudík 2013). Compared to the Maxwellian distribution, the ionization peak can be shifted up to $\Delta \log (T / \mathrm{K}) \approx 0.15$ for $\kappa=2$. Therefore, the Fe IX - Fe XIII line intensities are expected to peak at higher $\log (T / \mathrm{K})$ if $\kappa=2-3$.

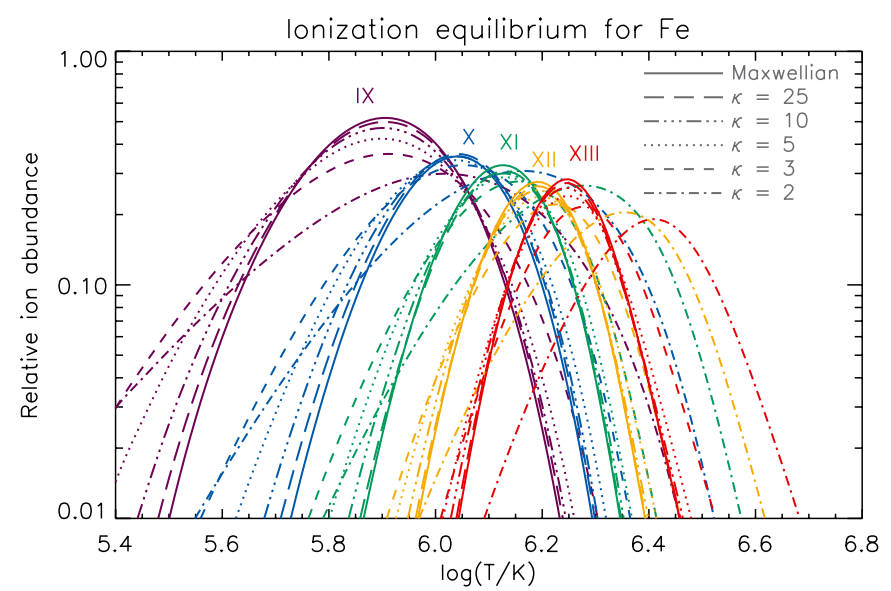

Fig. 2. Ionization equilibrium for the $\kappa$-distributions. Relative ion abundances for Fe IX-Fe XIII are plotted as a function of $T$ for $\kappa=2,3,5$, 10, 25, and the Maxwellian distribution.

\subsection{Excitation rates and excitation equilibrium}

The relative level populations, described by the ratios $n\left(X_{j}^{+k}\right) / n\left(X^{+k}\right)$ are obtained here under the assumption of an equilibrium situation. This assumption means that the total number of transitions from the level $j$ to any other level $m$ must be balanced by the total number of transitions from the other levels $m$ to the level $j$. In coronal conditions, the transitions are due to collisional excitation and de-excitation by an impacting electron, as well as spontaneous radiative decay. If we denote the rates of the collisional excitation and de-excitation as $C_{j m}^{\mathrm{e}}$ and $C_{j m}^{\mathrm{d}}$, the excitation equilibrium can be obtained by solving the following equations for each level $j$,

$$
\begin{array}{r}
\sum_{j>m} n\left(X_{j}^{+k}\right) n_{\mathrm{e}} C_{j m}^{\mathrm{d}}+\sum_{j<m} n\left(X_{j}^{+k}\right) n_{\mathrm{e}} C_{j m}^{\mathrm{e}}+\sum_{j>m} n\left(X_{j}^{+k}\right) A_{j m} \\
=n\left(X_{j}^{+k}\right)\left(\sum_{j<m} n_{\mathrm{e}} C_{m j}^{\mathrm{d}}+\sum_{j>m} n_{\mathrm{e}} C_{m j}^{\mathrm{e}}+\sum_{j<m} A_{m j}\right),
\end{array}
$$

where the left-hand side of Eq. (4) contains transitions $j \rightarrow m$, and the right-hand side contains transitions $m \rightarrow j$. Equation (4) must be supplemented with the condition that

$\sum_{j} n\left(X_{j}^{+k}\right)=n\left(X^{+k}\right)$

The electron collisional excitation rate for the upward transition $i \rightarrow j$ is given by the expression

$C_{i j}^{\mathrm{e}}(T, \kappa)=\sqrt{\frac{2}{m_{\mathrm{e}}}} \frac{\pi a_{0}^{2}}{\omega_{i}} I_{\mathrm{H}} \int_{0}^{+\infty} \Omega_{i j}\left(E_{i}\right) E_{i}^{-1 / 2} f_{\kappa}\left(E_{i}\right) \mathrm{d} E_{j}$,

where $a_{0}$ is the Bohr radius, $m_{\mathrm{e}}$ is the electron mass, $I_{\mathrm{H}} \approx$ $13.6 \mathrm{eV} \equiv 1 \mathrm{Ryd}$ is the hydrogen ionization energy, $\omega_{i}$ is the statistical weight of the level $i, E_{i}$ is the incident electron energy energy, and $E_{j}=E_{i}-\Delta E_{j i}$ is the final electron energy. The quantity $\Omega_{j i}\left(E_{j}\right)=\Omega_{i j}\left(E_{i}\right)$ is the collision strength (nondimensionalized cross section), given by

$\Omega_{j i}\left(E_{j}\right)=\omega_{j} \frac{E_{j}}{I_{\mathrm{H}}} \frac{\sigma_{j i}^{\mathrm{d}}\left(E_{j}\right)}{\pi a_{0}^{2}}=\omega_{i} \frac{E_{i}}{I_{\mathrm{H}}} \frac{\sigma_{i j}^{\mathrm{e}}\left(E_{i}\right)}{\pi a_{0}^{2}}$, 
where the $\sigma_{j i}^{\mathrm{e}}$ and $\sigma_{i j}^{\mathrm{d}}$ are the cross sections for the electron impact excitation and de-excitation, respectively. The collisional de-excitation is essentially a reverse process, so that

$$
C_{j i}^{\mathrm{d}}(T, \kappa)=\sqrt{\frac{2}{m_{\mathrm{e}}}} \frac{\pi a_{0}^{2}}{\omega_{j}} I_{\mathrm{H}} \int_{0}^{+\infty} \Omega_{j i}\left(E_{j}\right) E_{j}^{-1 / 2} f_{\kappa}\left(E_{j}\right) \mathrm{d} E_{i} .
$$

\subsection{Collision strengths datasets}

In this paper, we use the collision strength data obtained by R-matrix calculations (Badnell 1997, 2011). The individual atomic data were calculated by the APAP team ${ }^{1}$ for Fe IX, Fe X, Fe XII, and Fe XIII by Del Zanna et al. (2014), Del Zanna et al. (2012a), Del Zanna et al. (2012b), and Del Zanna \& Storey (2012), respectively. For Fe XI, the atomic data of Del Zanna \& Storey (2013) are used, except transitions involving levels 37, 39 , and 41 ( $J=1$ levels in the $3 \mathrm{~s}^{2} 3 \mathrm{p}^{3} 3 \mathrm{~d}$ configuration), for which the target failed to provide accurate energies and collision strengths. We use the earlier atomic data of Del Zanna et al. (2010) for the transitions involving these three levels. This has to be done, because using incorrect collision strengths for transitions that have strong observed intensities (see Del Zanna et al. 2010, Table 2 therein) will affect the calculated intensities of other lines through Eq. (4).

An example of the collision strength for the Fe XI $257.57 \AA$ line is provided in Fig. A.1, top right. Typically, the collision strengths are calculated in non-uniform grid of incident energy $E_{i}$ that covers all of the excitation tresholds and resonance regions. It spans from $E_{i}=0$ up to several tens of Ryd as needed, and contains up to ten thousand $E_{i}^{(q)}$ points. The high-energy $\operatorname{limit}\left(E_{i} \rightarrow+\infty\right)$ is also provided.

The $A_{j i}$ values required to calculate the relative level populations (Eq. (4)) for each ion are also provided (Del Zanna et al. 2014, 2012a; Del Zanna \& Storey 2013; Del Zanna et al. 2010, 2012b; Del Zanna \& Storey 2012).

\subsection{Calculation of the distribution-averaged collision strengths for $\kappa$-distributions}

It is advantageous to define the generalized distributionaveraged collision strengths Upsilon and Downsilon, $\Upsilon_{i j}(T, \kappa)$ and $\boldsymbol{J}_{j i}(T, \kappa)$ (Bryans 2006)

$\Upsilon_{i j}(T, \kappa)=\frac{\sqrt{\pi}}{2} \exp \left(\frac{\Delta E_{j i}}{k_{\mathrm{B}} T}\right) \int_{0}^{+\infty} \Omega_{i j}\left(E_{i}\right)\left(\frac{E_{i}}{k_{\mathrm{B}} T}\right)^{-\frac{1}{2}} f_{\kappa}\left(E_{i}\right) \mathrm{d} E_{j}$

$\mathrm{J}_{j i}(T, \kappa)=\frac{\sqrt{\pi}}{2} \int_{0}^{+\infty} \Omega_{j i}\left(E_{j}\right)\left(\frac{E_{j}}{k_{\mathrm{B}} T}\right)^{-\frac{1}{2}} f_{\kappa}\left(E_{j}\right) \mathrm{d} E_{i}$,

so that the collision excitation and de-excitation rates are given by

$C_{i j}^{\mathrm{e}}(T, \kappa)=\left(\frac{2 \pi}{m_{\mathrm{e}} k_{\mathrm{B}} T}\right)^{1 / 2} \frac{2 a_{0}^{2}}{\omega_{i}} I_{\mathrm{H}} \exp \left(-\frac{\Delta E_{j i}}{k_{\mathrm{B}} T}\right) \Upsilon_{i j}(T, \kappa)$,

$C_{j i}^{\mathrm{d}}(T, \kappa)=\left(\frac{2 \pi}{m_{\mathrm{e}} k_{\mathrm{B}} T}\right)^{1 / 2} \frac{2 a_{0}^{2}}{\omega_{j}} I_{\mathrm{H}} \mathrm{J}_{j i}(T, \kappa)$.

\footnotetext{
1 http://www . apap-network . org
}

After substituting Eq. (1) to Eqs. (9) and (10), the expressions for $\Upsilon_{i j}(T, \kappa)$ and $\boldsymbol{J}_{j i}(T, \kappa)$ can be written as

$$
\begin{aligned}
& \Upsilon_{i j}(T, \kappa)=A_{\kappa} \exp \left(\frac{\Delta E_{j i}}{k_{\mathrm{B}} T}\right) \int_{0}^{+\infty} \frac{\Omega_{j i}\left(E_{j}\right)}{\left(1+\frac{E_{j}+\Delta E_{j i}}{(\kappa-3 / 2) k_{\mathrm{B}} T}\right)^{\kappa+1}} \mathrm{~d}\left(\frac{E_{j}}{k_{\mathrm{B}} T}\right), \\
& \mathrm{J}_{j i}(T, \kappa)=A_{\kappa} \int_{0}^{+\infty} \frac{\Omega_{j i}\left(E_{j}\right)}{\left(1+\frac{E_{j}}{(\kappa-3 / 2) k_{\mathrm{B}} T}\right)^{\kappa+1}} \mathrm{~d}\left(\frac{E_{j}}{k_{\mathrm{B}} T}\right) .
\end{aligned}
$$

These quantities can then be used to calculate the corresponding excitation and de-excitation rates for $\kappa$-distributions (Eqs. (6) and (8)) in a similar manner to the $\Upsilon_{i j}(T)$ commonly used for the Maxwellian distribution (Seaton 1953; Burgess \& Tully 1992; Mason \& Monsignori Fossi 1994; Bradshaw \& Raymond 2013), for which the equality $\Upsilon_{i j}(T) \equiv \mathrm{J}_{j i}(T)$ holds.

In the CHIANTI database (Dere et al. 1997; Landi et al. 2013), the $\Upsilon$ 's are fed directly to the pop_solver.pro routine used to calculate the relative level populations. The pop_solver.pro routine utilizes a matrix solver in conjunction with the supplied collisional and radiative rates to calculate the relative level populations $n\left(X_{j}^{+k}\right) / n\left(X^{+k}\right)$. This routine was modified for $\kappa$-distributions in conjunction with the $\Upsilon$ s and Js obtained by numerical calculation of Eqs. (13) and (14). Excitation by protonion collisions was not taken into account, as it is negligible compared to the excitation by electron-ion collisions.

The numerical calculation of $\Upsilon_{i j}(T, \kappa)$ proceeds as follows. For each transition, the integral is approximated by the sum over the $N$ invididual energy points $E_{j}^{(q)}$ at which the collision strength $\Omega_{j i}\left(E_{j}\right)$ is calculated $(0 \leqq q \leqq N-1)$. A substitution $u=\left(E_{j}+\Delta E_{j i}\right) /\left((\kappa-3 / 2) k_{\mathrm{B}} T\right)$ is then performed. Next, following Burgess \& Tully (1992) and Bryans (2006), the $\Omega_{j i}(u)$ is approximated by a straight line between points $u\left(E_{j}^{(q)}\right)$ and $u\left(E_{j}^{(q+1)}\right)$, so that

$\Omega_{j i}\left(u^{(q)} \leqq u \leqq u^{(q+1)}\right)=w_{1}^{(q)} u+w_{0}^{(q)}$,

where $w_{0,1}^{(q)}$ are constants within the energy interval $E_{j}^{(q)} \leqq E_{j} \leqq E_{j}^{(q+1)}$. Finally, Eq. (13) is analytically integrated to obtain

$$
\begin{aligned}
& \Upsilon_{i j}(T, \kappa)=A_{\kappa}(\kappa-3 / 2) \exp \left(\frac{\Delta E_{j i}}{k_{\mathrm{B}} T}\right) \\
& \times \sum_{q=0}^{N-1}\left[-\frac{1}{\kappa}\left(w_{1}^{(q)} u+w_{0}^{(q)}\right)(1+u)^{-\kappa}-\frac{w_{1}^{(q)}}{\kappa(\kappa-1)}(1+u)^{1-\kappa}\right]_{E_{j}^{(q)}}^{E_{j}^{(q+1)}}
\end{aligned}
$$

The $\mathrm{J}_{j i}(T, \kappa)$ can be obtained in a similar manner.

The high-energy limit $E_{j} \rightarrow+\infty$ is in the numerical calculations set to $10^{5} \mathrm{Ryd}$. Between the last energy point $E_{j}$ and the value of $E_{j}=10^{5} \mathrm{Ryd}, \Omega_{j i}$ is approximated by a straight line in the scaled Burgess \& Tully (1992) domain. We note that the type of the scaling depends on the type of the transition (Burgess \& Tully 1992). Additional energy points are added to fill the space between the last $E_{j}^{(q)}$ point and the $E_{j}=10^{5} \mathrm{Ryd}$. We found that about $\approx 10^{3}$ points are required. We tested that the $\Upsilon_{i j}(T, \kappa)$ and $\mathrm{J}_{j i}(T, \kappa)$ calculated are not sensitive either to the choice of the high-energy limit or to the number of additional points. This is because the sub-integral expression in Eqs. (13) and (14) decreases steeply with $E_{j}$ for any $\kappa>3 / 2$. 


\section{Synthetic spectra for Fe IX-Fe XIII}

Having obtained the $\Upsilon_{i j}(T, \kappa)$ and $\boldsymbol{J}_{j i}(T, \kappa)$ for $\kappa$-distributions, it is straightforward to calculate the synthetic spectra for Fe IXFe XIII. In such calculations, we take into account transitions from all upper levels to only several tens of lower levels $i$. This is done in order to limit the size of calculations to several $\mathrm{GiB}$, while retaining all potentially observable transitions together with their selfblends. The number of lower levels is chosen so that all of the metastable levels with relative populations greater than $10^{-3}$ at densities of up to $\log \left(n_{\mathrm{e}} / \mathrm{cm}^{-3}\right)=12$ are retained, as well as to account for contributions to excitation of metastable levels by cascading from higher levels (e.g. Del Zanna \& Storey 2013, Sect. 4.2). This results in calculating the transitions from all upper levels to the lower 80 levels for Fe IX, lower 100 levels for Fe X, 32 for Fe XI, and 25 for both Fe XII and Fe XIII. An exception is the mestatable level 317 for Fe IX $\left(3 s^{2} 3 p^{3} 3 d^{3}{ }^{7} K_{8}\right)$, which can have a relative population of $\approx 10^{-3}$ for $\log \left(n_{\mathrm{e}} / \mathrm{cm}^{3}\right)>11$ and a Maxwellian distribution. The relative population of this level decreases with $\kappa$ and we chose not to include transitions populated from this level to limit the size of the calculations, so that they could be performed on a desktop PC. Then, the total number of resulting transitions calculated are $\approx 3.38 \times 10^{5}$ for Fe IX, $1.48 \times 10^{5}$ for Fe X, $8.2 \times 10^{4}$ for Fe XI, $4 \times 10^{5}$ for Fe XII, and $5.4 \times 10^{4}$ for Fe XIII. However, the vast majority of these transitions are extremely weak and unobservable.

The calculations of the synthetic spectra are performed for a temperature grid of $5 \leqq \log (T / \mathrm{K}) \leqq 7$ with a step of $\Delta \log (T / \mathrm{K})=0.025$. The range of electron densities is $8 \leqq$ $\log \left(n_{\mathrm{e}} / \mathrm{cm}^{-3}\right) \leqq 12$ covering a range of astrophysical plasmas in collisional ionization equilibrium, in particular the solar and stellar coronae.

In Fig. 3 (left), the Fe IX-Fe XIII synthetic spectra are plotted for an emission measure of unity and wavelengths $\lambda$ between $10^{2} \AA$ and $2 \times 10^{4} \AA$. The wavelength resolution of these spectra is $\Delta \lambda=1 \AA$. The electron density chosen is $\log \left(n_{\mathrm{e}} / \mathrm{cm}^{-3}\right)=9$, while the selected values of $\log (T / \mathrm{K})$ for each ion correspond to the peak of the respective ion abundance for the Maxwellian distribution. The changes in spectra with $\kappa$-distributions are illustrated by overplotting the spectra for $\kappa=5$ (green) and 2 (red color). Typically, the spectra are dominated by EUV transitions between $\approx 150 \AA$ and $400 \AA$, with few strong forbidden transitions in the UV, visible, or infrared parts of the electromagnetic spectrum. We note that these forbidden transitions disappear at higher densities because of their low $A_{j i}$ (see Eq. (4)).

The general behaviour of the EUV lines is that their intensities decrease with $\kappa$, i.e. an increasing departure from the Maxwellian distribution (Fig. 3). Although the line intensities in Fig. 3 are plotted for $\log (T / K)$ corresponding to the maximum respective ion abundance for the Maxwellian distribution, this behaviour is general. A few exceptions occur, however, if the spectra are compared with spectra for e.g. $\kappa=2$ at higher $\log (T / \mathrm{K})$ corresponding to the maximum ion abundance for such $\kappa$ (see Fig. 2). In contrast, several of the forbidden lines show a reversed behaviour with $\kappa$ even at the same $T$. The best example is the Fe X $6376.26 \AA$ the red line in the optical wavelength range (Fig. 3, second row, left). Other forbidden lines do show decreases of intensity with $\kappa$, albeit much weaker than the EUV lines. An example is the Fe XI $7894 \AA$ infrared line or the Fe XII $2406 \AA$ (Fig. 3, left, rows 3-4). Figure 3, right, shows the intensity ratios (in photon units) of some of the forbidden transitions to the strongest EUV transition as a function of $\kappa$. The line intensities for the Maxwellian, $\kappa=5$ and 2 and temperatures corresponding to the maximum of the ionization peak for the Maxwellian distribution are listed in Tables B.1-B.5.

This behaviour of forbidden lines with $\kappa$ shows that the $\kappa$-distributions can contribute to the observed enhanced intensities of these lines compared to the EUV ones, reported from eclipse observations (Habbal et al. 2013, Figs. 2-4). The forbidden lines are observed up to $\approx 2 R_{\odot}$. Although the enhanced excitation of the forbidden lines may be to some degree a result of photoexcitation by photospheric radiation (Habbal et al. 2013), the enhancement of the Fe X 6376.26 A line occurs especially in less dense regions of the corona, such as in a coronal hole or at the boundary of a helmet streamer. Both these regions contain predominantly open magnetic field lines, and thus are source regions of the fast and slow solar wind, respectively. We note that especially the fast solar wind exhibits $\kappa$-distributions (e.g. Maksimovic et al. 1997a,b; Le Chat et al. 2011).

\section{Electron density diagnostics independent of $\kappa$}

Rhe line contribution function $G_{X, j i}\left(T, n_{\mathrm{e}}, \kappa\right)$ (see Eq. (2)) is a function of all three plasma parameters. Determining electron density from observations prior to and independently of $T$ and $\kappa$ would greatly simplify the plasma diagnostics (Dzif̌́áková \& Kulinová 2010; Mackovjak et al. 2013). Therefore, we searched preferentially for density-sensitive line ratios that are not strongly sensitive to $T$ and $\kappa$. Such line ratios have to contain lines belonging to the same ion in order to avoid strong sensitivity to $T$ and $\kappa$ coming from the relative ion abundance term $n\left(X^{+k}\right) / n(X)$ in Eq. (2).

\subsection{Line selection procedure and selfblends}

Since the calculated synthetic spectra contain tens of thousands of transitions (Sect. 5), the vast majority of which are very weak and unobservable, we performed the search only on the strong observable lines. We consider a line as observable if its intensity is at least $0.05 I_{\max }$, where $I_{\max }$ is the intensity of the strongest line produced by the same ion. In order to take into account changes in the spectra produced by electron density (Fig. 3, Sect. 5), we include lines having $I \geqq 0.05 I_{\max }$ at either $\log \left(n_{\mathrm{e}} / \mathrm{cm}^{-3}\right)=8$ or 12 , or both. For Fe IX, a value of $0.02 I_{\max }$ is chosen instead of the $0.05 I_{\max }$. This is due to the very strong intensity of the $171.073 \AA$ bright line, which in turn would lead to dismissing most of the Fe IX lines observed by EIS, notably the $197.862 \AA$ (first identified by Young 2009).

The selected observable lines also include selfblending transitions originating within a given ion. A weaker transition is considered to be a selfblend if its wavelength $\lambda$ is located within $50 \mathrm{~m} \AA$ of the stronger transition, and if its intensity is at least $5 \times 10^{-4} I_{\max }$. Although these values are arbitrary, they have been chosen to correspond to the typical wavelength resolution of EUV spectrometers (e.g. $47 \mathrm{~m} \AA$ at $185 \AA$ for Hinode/EIS, Culhane et al. 2007), as well as to limit the number of selfblending transitions only to the ones with a relevant intensity contribution.

The selection procedure including the selfblends is run automatically on synthetic spectra calculated for the Maxwellian distribution. Subsequently, the same lines are picked from the synthetic spectra calculated for the $\kappa$-distributions. This can be done, since there are no lines which are strong for a $\kappa$-distribution but unobservable for the Maxwellian distribution (Fig. 3). After the selection procedure, we are left with 30 observable lines of Fe IX, 20 for Fe X, 40 for Fe XI, and 31 for Fe XII as well 
A\&A 570, A124 (2014)
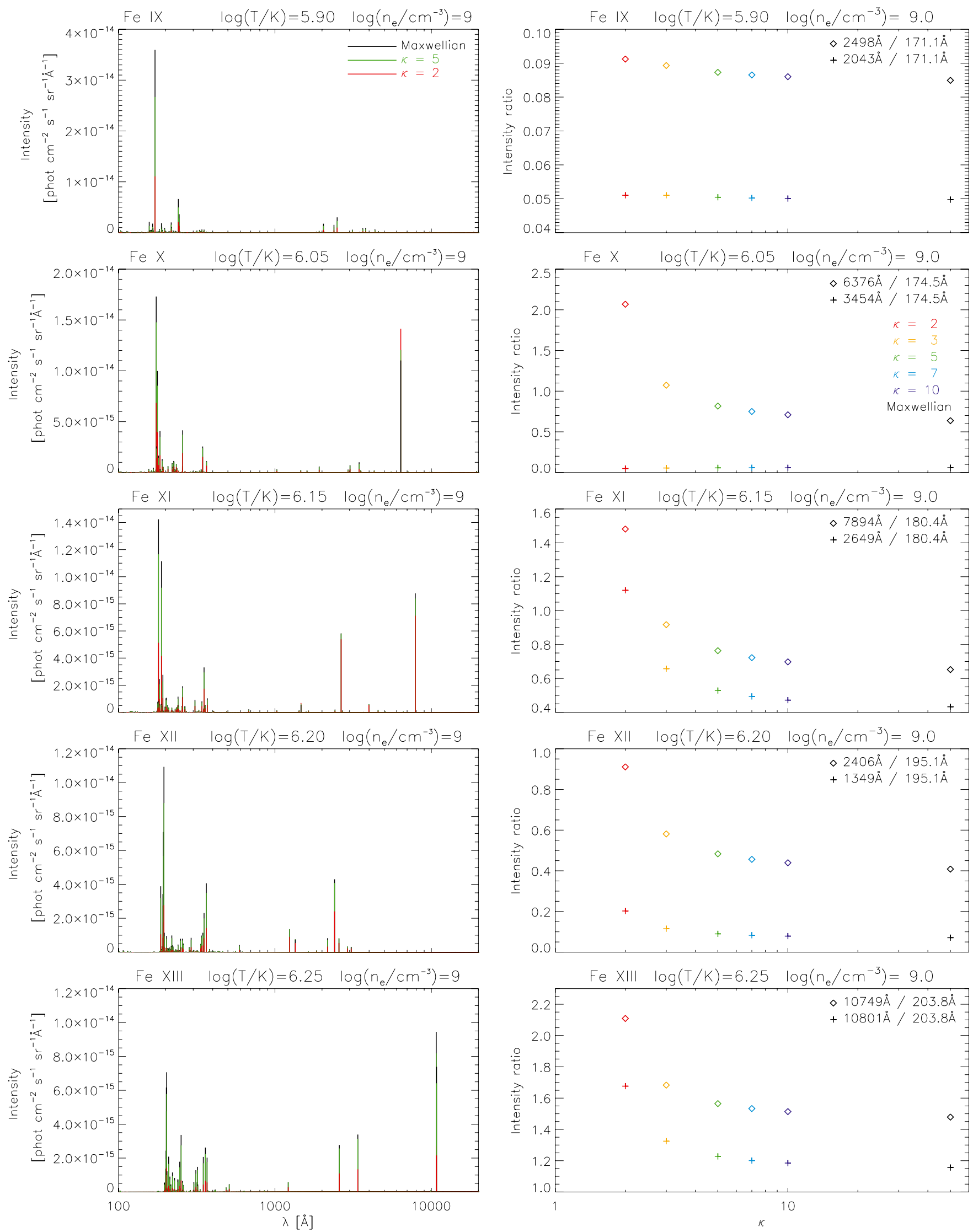

Fig. 3. Fe IX-Fe XIII spectra between $100 \AA$ and $20000 \AA$. The spectra shown have a resolution of $1 \AA$ and are shown for temperatures $T$ corresponding to the peak of the relative ion abundance for the Maxwellian distribution. Left: $\operatorname{spectra}$ for $\log \left(n_{\mathrm{e}}\right)=9 ;$ right: $\log \left(n_{\mathrm{e}}\right)=12$. 
J. Dudík et al.: Signatures of the non-Maxwellian $\kappa$-distributions in optically thin line spectra
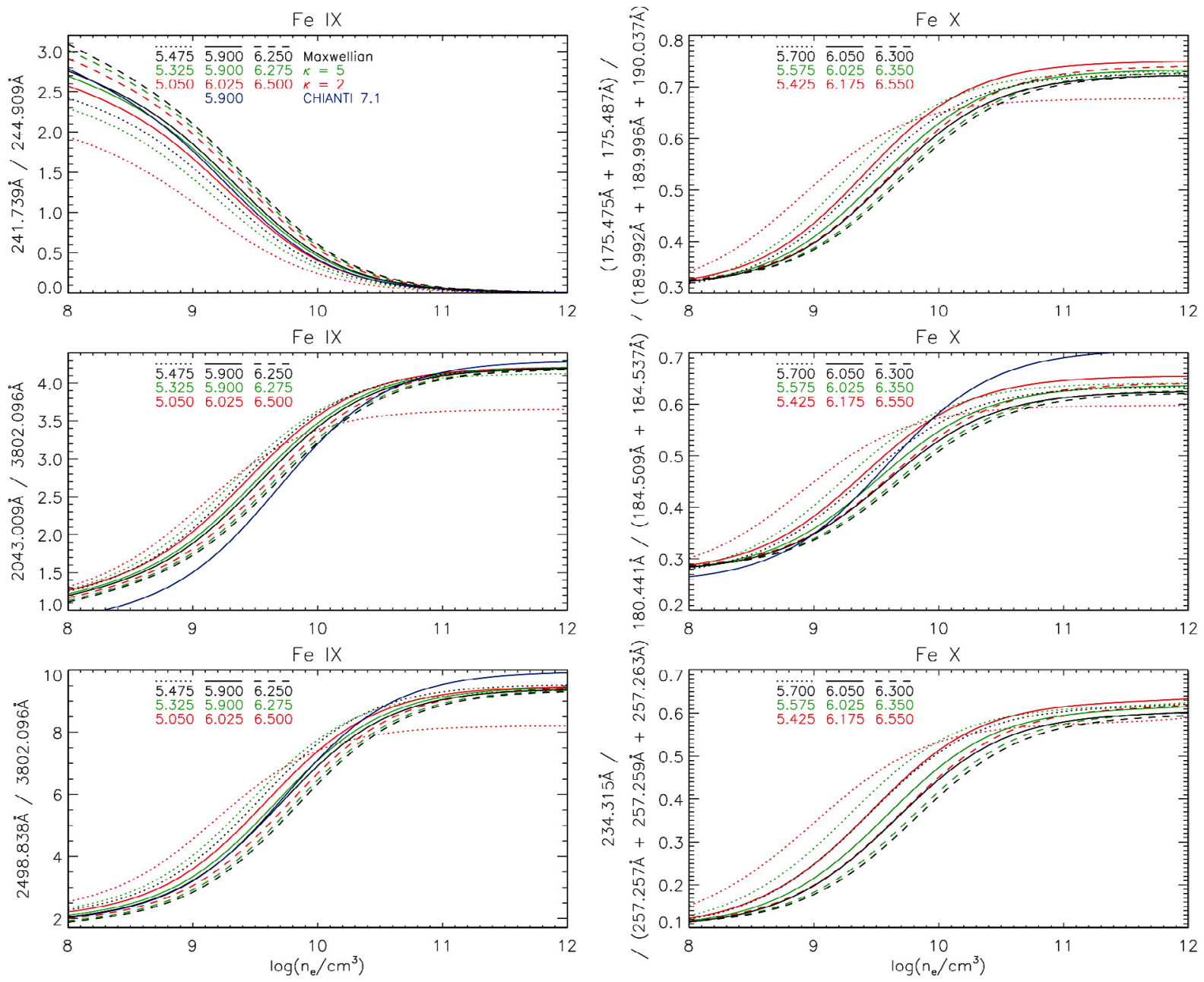

Fig. 4. Electron density diagnostics from Fe IX and Fe X. Intensity ratios of two lines are shown. The intensity units are phot $\mathrm{cm}^{-2} \mathrm{~s}^{-1} \mathrm{sr}^{-1}$. Black lines correspond to the Maxwellian distribution, green to $\kappa=5$, and red to $K=2$. Individual line styles are used to denote the $T$-dependence of the density diagnostics. Blue lines denote the same ratio according to the atomic data from CHIANTI v7.1.

as Fe XIII. The lines together with their selfblends are listed in Tables B.1-B.5. There, possible presence of blends from transitions in other ions are indicated as well.

\subsection{Line ratios recommended for density-diagnostics independently of $\kappa$}

We identify the best density diagnostics as line ratios that are both strongly dependent on $n_{\mathrm{e}}$ and relatively independent of $T$ and $\kappa$. The recommended ratios for density diagnostics are shown in Figs. 4-7. There, density-sensitive ratios of two lines are plotted as a function $n_{\mathrm{e}}$ for the Maxwellian distribution (black lines) together with $\kappa=5$ (green) and 2 (red lines). Blue lines correspond to the intensity ratios according to the CHIANTI v7.1 (Landi et al. 2013).

For the present calculations, the ratios are plotted at three different temperatures. These are the temperature of the peak of the relative ion abundance for the respective distribution (full lines), as well as temperatures corresponding to the $\approx 1 \%$ of the maximum of the relative ion abundance. We note that $1 \%$ of the relative ion abundance is an extreme value; usually, it is expected that the ion is formed at temperatures much closer to the peak of the ion abundance. However, this allows us to capture the sensitivity of the ratios to $T$ in the entire temperature range corresponding to the formation of each ion. It also provides an estimate of the maximum uncertainty of the diagnosed $\log \left(n_{\mathrm{e}} / \mathrm{cm}^{-3}\right)$ if the simultaneous diagnostics of $T$ and $\kappa$, described in Sect. 6 are not performed.

In the following, we report on density-sensitive line ratios throughout the electromagnetic spectrum. However, the discussion will be more focused on the line ratios observed by the Hinode/EIS, since this is a recent spectroscopic instrument with a large observed dataset.

\subsubsection{FeIX}

The ion Fe IX has only a few UV and EUV lines whose ratios can be used to determine $n_{\mathrm{e}}$. For line identifications, see e.g. Del Zanna et al. (2014) and references therein. The Fe IX 241.739 ̊/244.909 А (Storey et al. 2002; Del Zanna et al. $2014)$ is density-sensitive for $\log \left(n_{\mathrm{e}} / \mathrm{cm}^{3}\right) \lesssim 10$; however it has a non-negligible dependence on $T$ that increases for low $\kappa$ (Fig. 4, top left). We note that these lines are just outside of the EIS long-wavelength channel.

\subsubsection{Fe IX lines longward of $1000 \AA$}

The Fe IX ion also offers several density-sensitive ratios involving forbidden lines (Del Zanna et al. 2014). Two examples, the 

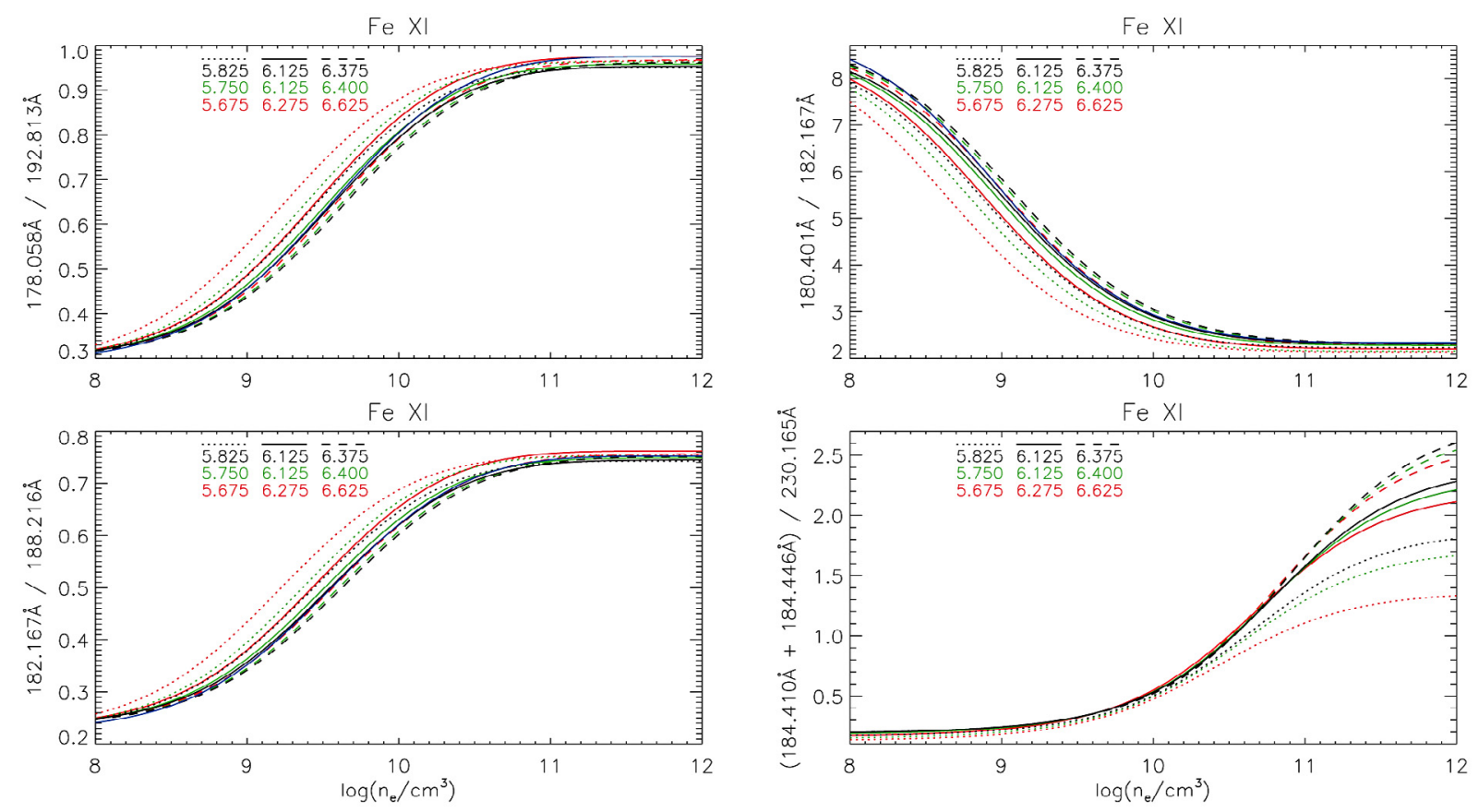

Fig. 5. Electron density diagnostics from Fe XI. Line styles and colors are the same as in Fig. 4.
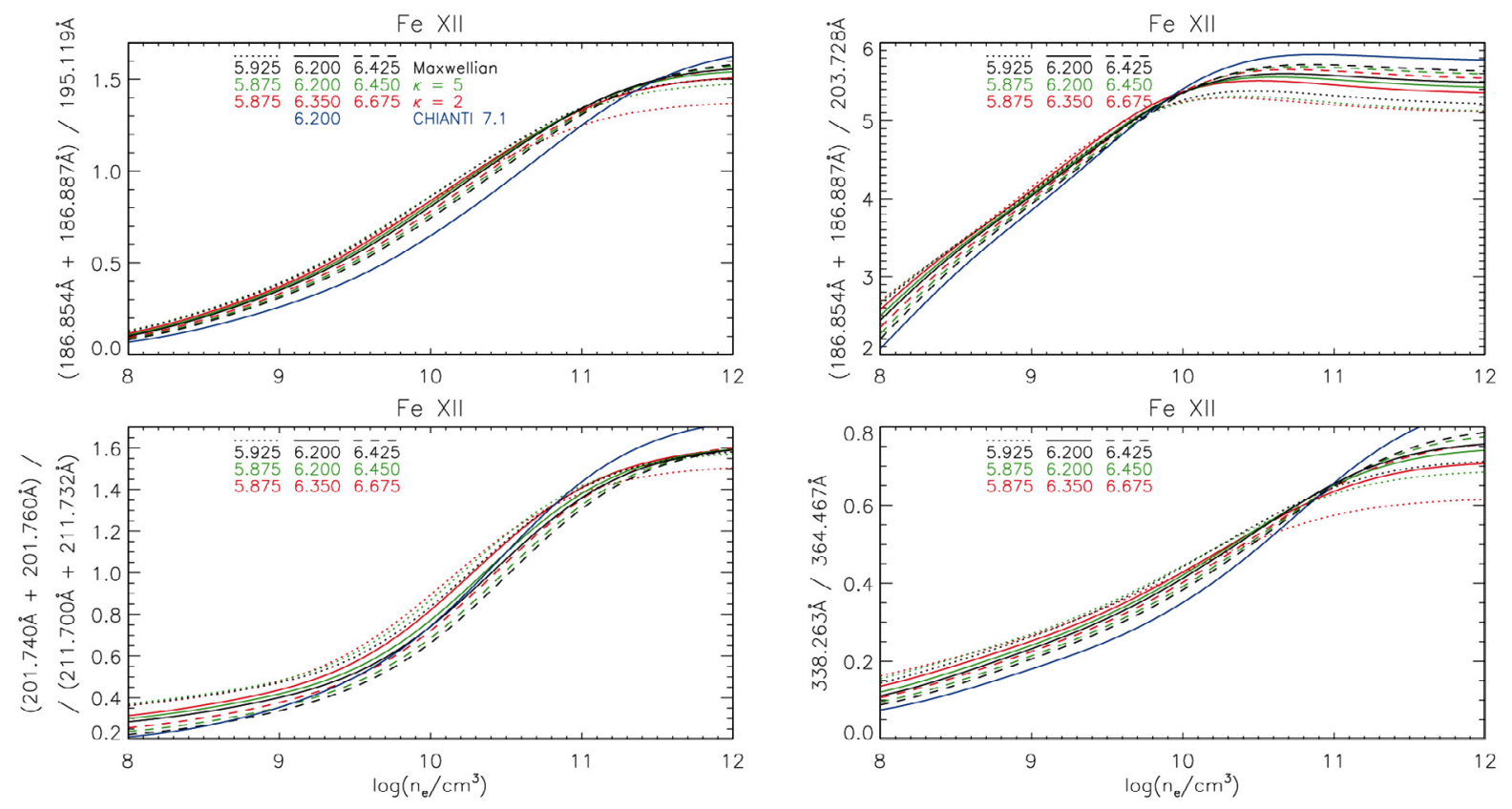

Fig. 6. Electron density diagnostics from Fe XII. Line styles and colors are the same as in Fig. 4.

$2043 \AA / 3802 \AA$ and $3644 \AA / 3802 \AA$ are shown in Fig. 4. The latter ratio shows a good agreement with the CHIANTI v7.1 atomic data (Fig. 4, left, bottom). Nevertheless, there are differences in present calculations for the Maxwellian distribution and the CHIANTI v7.1 atomic data, mainly for $\log \left(n_{\mathrm{e}} / \mathrm{cm}^{3}\right) \gtrsim 10$. The $2043 \AA$ line has different intensities for all densities, resulting in disagreement with the CHIANTI v7.1 calculations (Fig. 4, left, middle). We note that these line ratios also have non-negligible sensitivity to $T$.

\subsubsection{FeX}

For line identifications, see Del Zanna et al. (2004), Del Zanna et al. (2012a), and references therein. The Fe X ion has only a few density-sensitive line ratios that also show non-negligible sensitivity to $T$. All of these ratios are usable only for $\log \left(n_{\mathrm{e}} / \mathrm{cm}^{3}\right) \lesssim 10$. The three best ones are plotted in Fig. 4. For the $175.475 \AA / 190.037 \AA$ ratio, the sensitivity to $T$ increases with decreasing $\kappa$ (Fig. 4, top right). Both the lines involved are selfblended by transitions contributing $\approx 0.4-1.4 \%$, with the exception of $189.996 \AA$ transition, which contributes up to $2.7 \%$ at $\log \left(n_{\mathrm{e}} / \mathrm{cm}^{3}\right)=12$. We note that we do not show the corresponding ratio for the CHIANTI 7.1 atomic data due to differences in atomic data and numerous difficulties in identifying individual selfblending transitions.

The $180.441 \AA /(184.509 \AA+184.537 \AA)$ is also usable; however, the density sensitivity is rather weak. The $234.315 \AA /(257.257 \AA+257.259 \AA+257.263 \AA)$ shows 
J. Dudík et al.: Signatures of the non-Maxwellian $\kappa$-distributions in optically thin line spectra
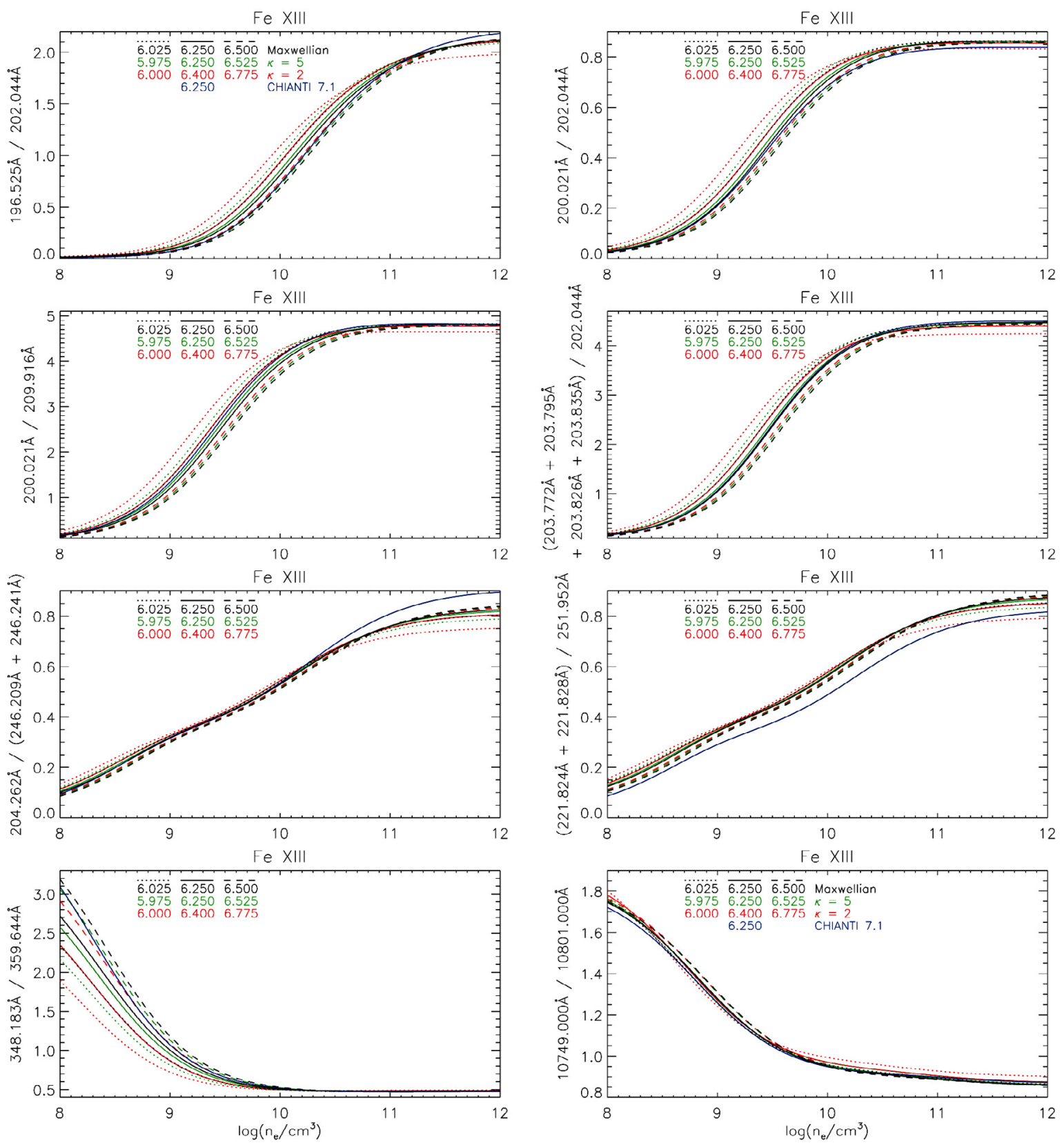

Fig. 7. Electron density diagnostics from Fe XIII. Line styles and colors are the same as in Fig. 4.

a strong density-sensitivity, but involves the $234.315 \AA$ line, which is unobservable by the EIS instrument. The selfblend at $257.3 \AA$ can be used to diagnose $n_{\mathrm{e}}$ also in conjuction with other lines, e.g. $207.449 \AA, 220.247 \AA$, $224.800 \AA, 225.856 \AA$, or $226.998 \AA$. We note that the selfblend at $257.3 \AA$ is dominated by the $257.263 \AA$ line at $\log \left(n_{\mathrm{e}} / \mathrm{cm}^{3}\right) \lesssim 8$, but at higher densities, the $257.259 \AA$ line is the dominant one. Finally, the $234.315 \AA$ line is not included in CHIANTI v7.1.

\subsubsection{FeXI}

For line identifications, see Del Zanna (2010, 2012 and references therein). The Fe XI ion offers several density-sensitive ratios (Fig. 5). Here we report only on those that are not strongly sensitive to $\kappa$. The $178.058 \AA / 192.813 \AA$, $180.401 \AA / 182.167 \AA$, and $182.167 \AA / 188.216 \AA$ are all usable at $\log \left(n_{\mathrm{e}} / \mathrm{cm}^{3}\right) \lesssim 10$. They show a weak dependence on $\kappa$, with $\kappa=2$ providing systematically $\operatorname{lower} \log \left(n_{\mathrm{e}}\right)$ by about 0.2 dex (Mackovjak et al. 2013). At higher densities, the $184.410 \AA / 230.165 \AA$ ratio can be used. This ratio shows very low dependence on $T$. However, it involves a $230.165 \AA$ line not observable by Hinode/EIS. The $184.410 \AA$ line is blended by a $184.446 \AA$ transition contributing of up to $7 \%$ of the intensity, especially at low densities. We note that both the $184.446 \AA$ and $230.165 \AA$ lines are not included in CHIANTI 7.1.

\subsubsection{FeXII}

For line identifications, see Del Zanna \& Mason (2005), Del Zanna (2012), and references therein. The Fe XII also offers several density-sensitive ratios that are insensitive to $\kappa$ (Fig. 6). Two of these are the $(186.854 \AA+186.887 \AA) / 195.119 \AA$ and $(186.854 \AA+186.887 \AA) / 203.728 \AA$ ratios. The $186.887 \AA$ is the stronger line, with the $186.854 \AA$ having $20-60 \%$ of 
the $186.887 \AA$ intensity at $\log \left(n_{\mathrm{e}} / \mathrm{cm}^{3}\right)=8-12$, respectively. We note that these ratios are shifted to lower densities compared to the CHIANTI 7.1 results (Del Zanna et al. $2012 b$ ). We also note that the $203.728 \AA$ line is actually blending the stronger Fe XIII selfblend at $203.8 \AA$ (e.g. Young et al. 2009, Fig. 9 therein). Careful deblending should be performed if this line is to be used for density diagnostics. Other available Fe XII ratios include the $338.263 \AA / 364.467 \AA$ and the $(201.740 \AA+201.760 \AA) /(211.700 \AA+211.732 \AA)$. The $201.760 \AA$ has about $60-90 \%$ intensity of the $201.740 \AA$ line. On the other hand, the $211.700 \AA$ has only about $2-3 \%$ intensity compared to the $211.732 \AA$ line.

\subsubsection{Fe XIII}

For line identifications, see Del Zanna (2011a) and references therein. The Fe XIII has many density-sensitive line ratios, several of which are observable by Hinode/EIS (Young et al. 2009; Watanabe et al. 2009). In Fig. 7 we show the best ones. The $196.525 \AA / 202.044 \AA, 200.021 \AA / 202.044 \AA$, and $200.021 \AA / 209.916 \AA$ are all strongly density-sensitive and all contain unblended lines. The usually used $203.8 \AA / 202.044 \AA$ ratio involves a complicated selfblend at $203.8 \AA$ that contains four transitions, $203.772 \AA, 203.765 \AA, 203.826 \AA$, and $203.835 \AA$ (Del Zanna 2011a). The main contributor is the 203.835 $\AA$ line, with the contribution of other lines at $\log \left(n_{\mathrm{e}} / \mathrm{cm}^{3}\right)=8-12$ ranging from $\approx 23$ to $1 \%, 38-2 \%$, and $47-36 \%$ of the $203.835 \AA$ intensity, respectively. The $203.8 \AA / 202.044 \AA$ ratio saturates at $\approx 4.4$ for $\log \left(n_{\mathrm{e}} / \mathrm{cm}^{3}\right)=10.5$.

The $204.262 \AA /(246.209 \AA+246.241 \AA)$ and the $(221.824 \AA+221.828 \AA) / 251.952 \AA$ are also excellent density diagnostics, both having negligible dependence on both $T$ and $\kappa$. The intensity of the $246.241 \AA$ line is about $0.1-0.8 \%$ of the $246.209 \AA$ line, its contribution to the selfblend increasing with density. Similarly, the intensity of the $221.824 \AA$ is about $19-2 \%$ of the $221.828 \AA$ line, again strongly decreasing with density. The 249.241 $\AA$ and 221.824 $\AA$ lines are however absent from CHIANTI 7.1. There are also differences in our atomic data compared to the CHIANTI 7.1 for the $221.828 \AA$ line.

\subsubsection{Fe XIII lines longward of $1000 \AA$}

The pair of infrared lines at $10749.0 \AA$ and $10801.0 \AA$ also provide a good density diagnostic (e.g. Flower \& Pineau des Forets 1973; Wiik et al. 1994; Singh et al. 2002) (Fig. 7, bottom right). Here, the ratio is usable up to higher densities of up to $\log \left(n_{\mathrm{e}} / \mathrm{cm}^{3}\right) \approx 9.5$, compared to the original paper of Flower $\&$ Pineau des Forets (1973). These lines can be measured by spectrographs mounted on the ground-based coronagraphs, such as the Coronal Multichannel Polarimeter (COMP-S) instrument being installed at the Lomnicky Peak observatory (Tomczyk et al. 2007; Tomczyk \& McIntosh 2009; Schwartz et al. 2012, 2014).

\section{Theoretical diagnostics of $T$ and $\kappa$}

Once the electron density $n_{\mathrm{e}}$ is determined, $T$ and $\kappa$ can be diagnosed simultaneously using the ratio-ratio technique involving line ratios sensitive to both $\kappa$ and $T$ (Dzifčáková \& Kulinová 2010, 2011; Mackovjak et al. 2013). We note that these papers investigated specific Fe lines observable by EIS (Dzifčáková \& Kulinová 2010), non-Fe EIS lines (Mackovjak et al. 2013), and Si III observed by SUMER (Dzifčáková \& Kulinová 2011). Other wavelength ranges have not yet been investigated.

Here, we investigate the lines selected using the procedure described in Sect. 5.1 for sensitivity to $\kappa$-distributions using the ratio-ratio technique. The ratio-ratio diagrams are constructed using intensities of three lines, with $R_{X}=I_{1} / I_{2}$ and $R_{Y}=I_{3} / I_{1}$, where the wavelengths obey the relation $\lambda_{1}<\lambda_{2}<\lambda_{3}$. If the number of lines selected (Sect. 5.1) is $\mathcal{N}$, then the number of possible ratio-ratio diagrams, i.e. the $R_{Y}\left(R_{X}\right)$ combinations is given by $\mathcal{N} ! /(3 !(\mathcal{N}-3) !)$. We note that in principle, four different lines can be used, with $R_{Y}=I_{3} / I_{4}$. The number of possible ratioratio diagrams would then be increased by a factor of $(\mathcal{N}-3) / 4$. For the Fe XI this would increase the possible number of ratioratio diagrams from 9880 by a factor of 9.25 (see Sect. 5.1), which is impractical. We thus limited our investigation to the ratio-ratio diagrams using only intensities of three lines, with the additional restriction that the ratios of line intensities either larger than 20 or lower than 1/20 are not investigated except for Fe IX (Sect. 5.1), because with such different line intensities, the weaker line would have considerably more photon noise, limiting the usefulness of the diagnostics. Furthermore, ratio-ratio diagrams that exhibit sensitivity to $\kappa$ in only a very limited range of electron densities (less than an order of magnitude) are not considered here.

The ratio-ratio diagrams here are also limited to the lines emitted by the same ion (Dzifčáková \& Kulinová 2010; Mackovjak et al. 2013). This is done to avoid an additional source of errors coming from uncertainties in the ionization and recombination rates. Therefore, any sensitivity of the line ratios to $\kappa$ has to originate in the excitation processes alone.

In the following subsections, we report only on the ratios that show the highest sensitivity to $\kappa$. The vast majority of the possible $R_{Y}\left(R_{X}\right)$ combinations either do not show sensitivity to $\kappa$, or only a very low one. This is important, since the presence of many insensitive lines can be misleading when interpreting solar spectra, as the spectra can then be fitted simply with some values of $T$ and emission measure under the assumption of the Maxwellian distribution, without providing any clue to the nature of the distribution function of the emitting plasma.

\subsection{Fe IX}

This ion offers the best options for diagnosing $\kappa$ and $T$, since unlike the Fe X-Fe XIII, the transitions selected for Fe IX contain several that do not involve the first few energy levels (Tables B.1-B.5). The most conspicuous example is the 13-148 transition constituting a line at $197.862 \AA$.

Examples of the best diagnostics options are shown in Fig. 8. The $177.592 \AA / 171.073 \AA-189.941 \AA / 177.592 \AA$ has the lowest sensitivity to density, while having a high sensitivity to $\kappa$, by about a factor of 2 difference between $\kappa=2$ and the Maxwellian distribution. Strong sensitivity to $T$ is also present, mainly due to the $189.941 \AA / 177.592 \AA$ ratio. The $189.941 \AA / 197.862 \AA-317.193 \AA / 189.941 \AA$ and the $197.862 \AA / 171.073 \AA-189.941 \AA / 197.862 \AA$ ratio-ratio diagrams also have a strong sensitivity to $\kappa$, but in these cases the sensitivity decreases strongly with increasing $\log (T / \mathrm{K})$. The sensitivity of the $217.101 \AA / 244.909 \AA-329.897 \AA / 217.101 \AA$ to $\kappa$ decreases with increasing $n_{\mathrm{e}}$ and at $\operatorname{low} \log (T / \mathrm{K})$ is further complicated by the overlap of various $\kappa$. Nevertheless, this diagram still can be used for $\log (T / \mathrm{K}) \approx 5.8-6.1$. 
J. Dudík et al.: Signatures of the non-Maxwellian $\kappa$-distributions in optically thin line spectra
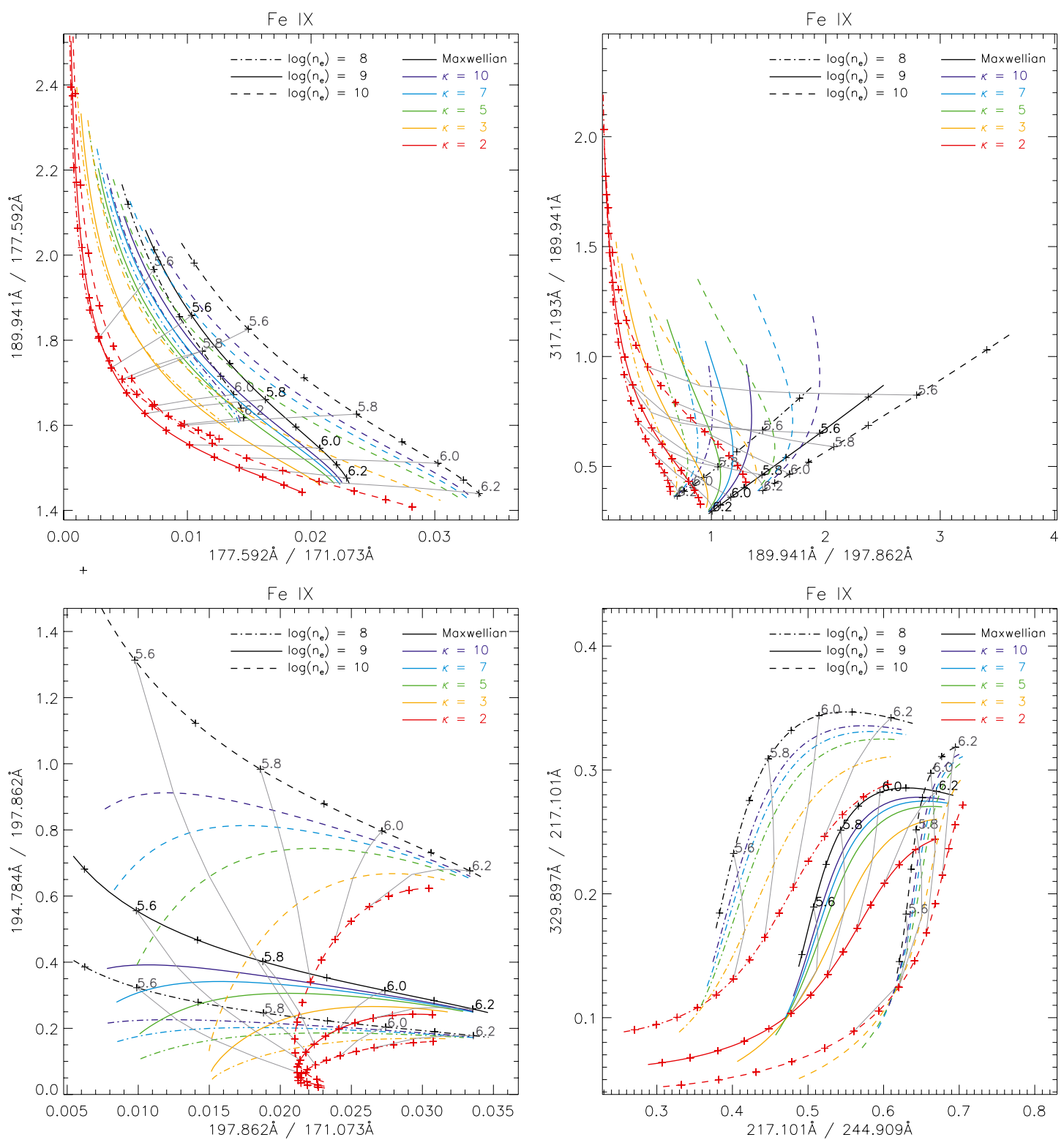

Fig. 8. Examples of the theoretical plots for simultaneous diagnostics of $T$ and $\kappa$ from Fe IX. Both axes show intensity ratios of two lines, with the line intensities in the units of phot $\mathrm{cm}^{-2} \mathrm{~s}^{-1} \mathrm{sr}^{-1}$. Individual line styles correspond to different $\log \left(n_{\mathrm{e}} / \mathrm{cm}^{3}\right)$, colors to individual values of $\kappa$, and gray lines denote isotherms connecting points having the same $\log (T / \mathrm{K})$.

The ratio-ratio diagrams presented here emphasize the need for independent diagnostics of the electron density (Sect. 5), which would in most cases complicate the diagnostics of $\kappa$.

\subsection{FeX}

The Fe $\mathrm{x}$ ion offers only a few possibilities of diagnosing $T$ and $\kappa$. Figure 9 (left) shows three examples. The first one is $180.441 \AA / 193.715 \AA-365.560 \AA / 180.441 \AA$. This ratioratio diagram exhibits sensitivity to density. The sensitivity to $\kappa$ comes mainly from the $365.560 \AA / 180.441 \AA$ ratio involving lines with different excitation thresholds. The sensitivity to $K$ is better at $\operatorname{lower} \log (T / \mathrm{K})$ and decreases towards higher values of $\log (T / \mathrm{K}) \approx 6.2$. The sensitivity to $T$ is given mainly by the $180.441 \AA / 193.715 \AA$ ratio. Other alternative ratios with similar sensitivities are, for example, the $180.441 \AA / 207.449 \AA-345.738 \AA / 180.441 \AA$, with the $345.738 \AA$ and the $365.560 \AA$ lines being formed from the same upper level (Table B.2), as well as the $(184.509 \AA+$ 184.537 $\AA) / 193.715 \AA-365.560 \AA /(184.509 \AA++184.537 \AA)$.

The second example shown in Fig. 9, left, middle is the $234.315 \AA / 256.398 \AA-345.738 \AA / 234.316 \AA$. We note that the sensitivity of these $\mathrm{Fe} \mathrm{X}$ ratios to $\kappa$ is again not much greater than a few tens of per cent. Such sensitivity occur for low $\kappa=2$ and decrease strongly with increasing $\log (T / \mathrm{K})$. We also note that this sensitivity is comparable to the typical calibration uncertainty of the EUV spectrometers (e.g. Culhane et al. 2007; Wang et al. 2011; Del Zanna 2013), which makes the diagnostics using these $\mathrm{Fe} \mathrm{X}$ lines unrealistic at present.

\subsubsection{FeX diagrams involving the $6376 \AA$ line}

The last example involves the red forbidden line at $6376.3 \AA$ (Fig. 9, left, bottom). The sensitivity to $\kappa$ comes from the $6376.3 \AA /(184.509 \AA+185.537 \AA)$, while the sensitivity 

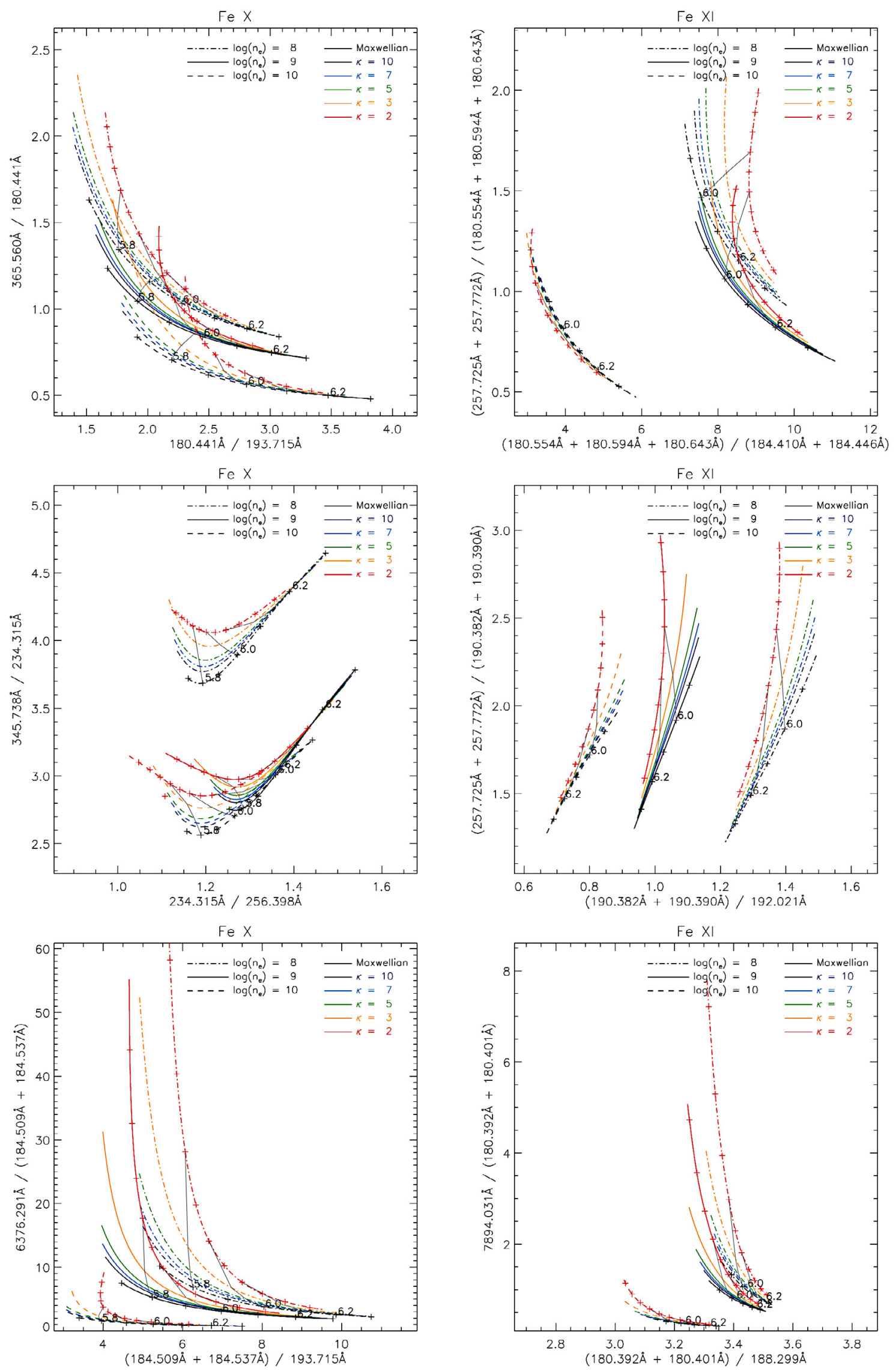

Fig. 9. Examples of the theoretical plots for simultaneous diagnostics of $T$ and $\kappa$ from Fe X (left) and Fe XI (right). Line styles and colors are the same as in Fig. 8. 
to $T$ arises from the $(184.509 \AA+185.537 \AA) / 193.715 \AA$ ratio. The overall shape is similar to Fig. 9, left, top. The sensitivity to $\kappa$ is greatest at $\operatorname{low} \log (T / \mathrm{K})$ and increases with decreasing density. This density-dependence of this ratios could make this diagram especially useful for low-density regions higher in the atmosphere, where the red line can still be strong.

\subsection{Fe XI}

The Fe XI ion has the largest number of observable lines (Sect. 5.1). It offers several options for simultaneous diagnostics of $T$ and $\kappa$. Here, we present several typical examples (Fig. 9, right. These ratio-ratio diagrams show that the sensitivity of individual line ratios to $\kappa$ is again typically only a few tens of per cent. In most instances, the magnitude of sensitivity depends on $\log (T)$, with greater sensitivities at lower temperatures as in the case of FeX.

The sensitivity to $T$ is obtained by using lines such as the selfblends at $257 \AA$ (Del Zanna et al. 2010), i.e. the $257.538 \AA+257.547 \AA+257.554 \AA+257.558 \AA$, dominated by the $257.547 \AA$ and $257.554 \AA$ lines; or the $257.725 \AA+$ $257.772 \AA$, dominated by the $257.772 \AA$ line. The theoretical diagrams presented here confirm that the ratios of these lines to lines at 178-211 $\AA$ (i.e. short-wavelength channel of the EIS instrument) are strongly sensitive to temperature. We note that other lines, such as the $234.730 \AA, 236.494 \AA, 239.780 \AA+$ $239.787 \AA, 308.554 \AA, 349.046 \AA, 356.519 \AA$, or $358.613 \AA$ can be used instead, depending on the particular combination with other lines in a given ratio-ratio diagram.

We note that some ratio-ratio diagrams can be used in a limited range of densities, for example, (180.554 $\AA+$ $180.594 \AA+180.643 \AA) /(184.410 \AA+180.446 \AA)-(257.725 \AA+$ $257.772 \AA) /(180.554 \AA+180.594 \AA+180.643 \AA$ ) (Fig. 9, right, top) can be used for $\log \left(n_{\mathrm{e}} / \mathrm{cm}^{3}\right) \approx 8-9$, i.e. at quiet Sun densities. Other ratios have similar sensitivity to $k$ independently of density. An example is the $(190.382 \AA+190.390 \AA) / 192.021 \AA-$ $(257.725 \AA+257.772 \AA) /(190.382 \AA+190.390 \AA)$, see Fig. 9 , right, middle. In this diagram, changes in $\kappa$ can be masked by relatively small changes in the electron density. This emphasizes the need for diagnosing $n_{\mathrm{e}}$ together with $T$ and $\kappa$, since some of the density-sensitive ratios presented in Sect. 5 have non-negiligible dependence on $T$.

\subsubsection{FeXI diagrams involving the $7894 \AA$ line}

The forbidden $7894.03 \AA$ line can be a strong indicator of departures from the Maxwellian distribution (see also Sect. 4 and Fig. 3). Its intensity can be enhanced several times relative to the EUV lines, e.g. $180.392 \AA+180.401 \AA$ (Fig. 9, bottom, right), which is the strongest EUV line of Fe XI. However, the enhancement decreases with electron density, and at $\log \left(n_{\mathrm{e}} / \mathrm{cm}^{3}\right) \approx 10$ is only a negligible one.

\subsection{Fe XII}

The Fe XII ion offers only a few opportunities for determining $\kappa$. The ratio-ratio diagrams are found to always be density-dependent, with only a very weak sensitivity to $\kappa$, of the order of several tens of per cent. Two typical examples, both involving forbidden lines, are shown in Fig. 10, top and middle. In these examples, the sensitivity to $\kappa$ originates from the $256.410 \AA / 346.852 \AA$ ratio. The $256.410 \AA / 346.852 \AA-$
1349.4 $\AA$ /256.410 ̊ involves the 1349.4 $\AA$ Fe XII line observable by the IRIS spectrometer (De Pontieu et al. 2014). This is a forbidden transition that shows temperature sensitivity when combined with EUV lines. The temperature sensitivity increases with $\kappa \rightarrow 2$ by more than a factor of two. Therefore, any anomalous intensities of this line could be caused by the presence of $\kappa$-distributions. We note that other forbidden lines, such as the $1242.01 \AA$ or the $2566.8 \AA$ can be used instead.

\subsection{Fe XIII}

The Fe XIII ion has very few line ratios sensitive to $\kappa$. The sensitivity is generally very weak and the ratio-ratio diagrams involve the $201.126 \AA$ line together with a forbidden transition in the near-ultraviolet. Examples include e.g. the $201.126 \AA / 261.743 \AA \approx-2579.540 \AA / 201.126 \AA$ (Fig. 10, bottom). A similar alternative is the $201.126 \AA / 239.030 \AA$ $3388.9 \AA / 201.126 \AA$, except that the $201.126 \AA / 239.030 \AA$ ratio increases monotonically with decreasing density. Similarly to the Fe XII case (Sect. 6.4), anomalous intensities of the forbidden lines can be a signature of the non-Maxwellian $\kappa$ distributions.

\section{Summary and discussion}

We performed a calculation of the distribution-averaged collision strengths for Fe IX-Fe XIII and subsequently the spectral synthesis for all wavelengths for the non-Maxwellian $\kappa$-distributions. We used the state-of-the art atomic data and searched for line ratios sensitive to electron density, temperature, and $\kappa$. In doing so, the previous exploratory work of Dzifčáková \& Kulinová (2010) was extended and superseded. We also investigated various collision strength approximations and their accuracy. The most important conclusions can be summarized as follows:

1. The calculated Fe IX-Fe XIII synthetic spectra show consistent behaviour with $\kappa$ for most of the EUV lines. Typically, the line intensities decrease with $\kappa$ at temperatures corresponding to the peak of the relative ion abundance for the Maxwellian distribution.

2. It is possible to perform a reliable diagnostics of the electron density without diagnostics of $\kappa$. Fe IX and Fe X offer only a few opportunities. The best densitydiagnostics that are not strongly sensitive to $T$ or $\kappa$ come from FeXI-Fe XIII. Some of the line ratios, such as the Fe XII $(186.854 \AA+186.887 \AA) / 195.119 \AA$, Fe XIII $204.262 \AA /(246.209 \AA+246.241 \AA)$, or the Fe XIII $10749.0 \AA / 10801.0 \AA$ show only a very weak dependence on $T$ and $\kappa$, and are excellent density diagnostics.

3. The consistent behaviour of EUV line intensities with $\kappa$ makes it is very difficult to perform diagnostics of $\kappa$ using only lines of one ion. The vast majority of line ratios have no sensitivity to $\kappa$ at all. A small number of line ratios exhibit sensitivity to $\kappa$. Typically, this sensitivity is of the order of only several tens of per cent, which is comparable to the calibration uncertainties of the present EUV instruments, such as the Hinode/EIS. Only very few line ratios, in particular for Fe IX, exhibit larger sensitivity. The best diagnostics options presented here often include lines unobservable by presentday EUV spectroscopic instrumentation.

4. Because of the above, signatures of the departures from the Maxwellian distribution will be inconspicuous in most of the observed EUV solar coronal spectra. In most instances, 

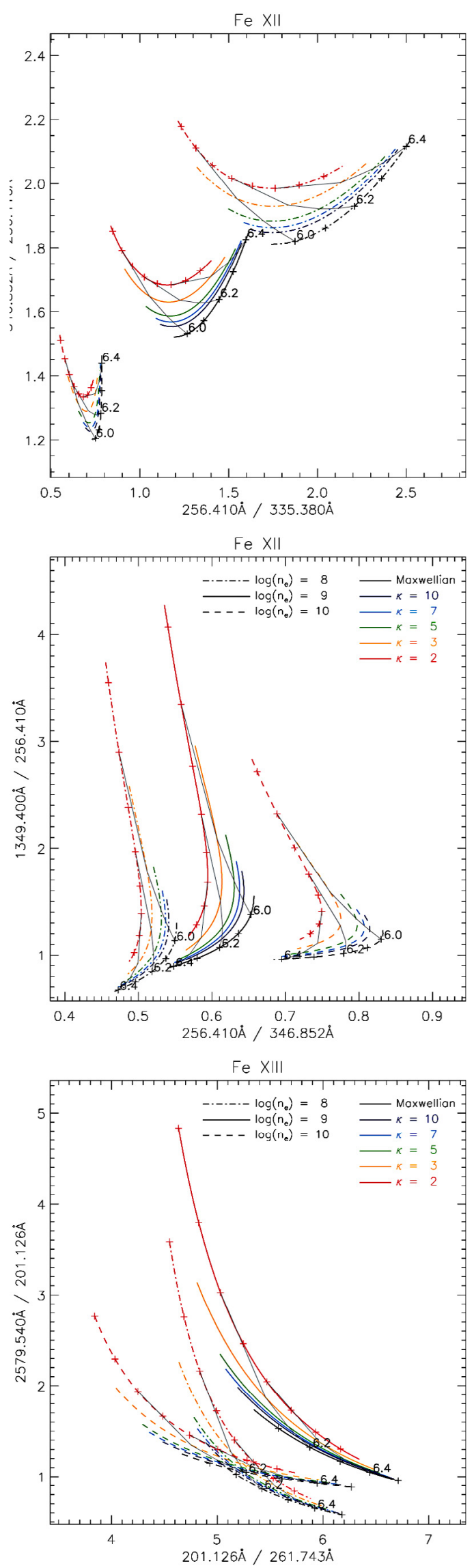

Fig. 10. Examples of the theoretical plots for simultaneous diagnostics of $T$ and $\kappa$ from Fe XII (top and middle), as well as Fe XIII (bottom). Line styles and colors are the same as in Fig. 8. small changes in electron density can obscure the changes in the spectra due to $\kappa$.

5. Several forbidden lines, such as the Fe X $6378.26 \AA$ line show reversed behaviour with $\kappa$. That is, the intensity of this line increases with decreasing $\kappa$. Other forbidden lines show decrease of intensity with $\kappa$; however, the decrease is not as strong as for the EUV lines. An example is the Fe XI $7894.03 \AA$ line whose intensity changes only weakly with $\kappa$. Therefore, these forbidden lines are a good indicator of the departures from the Maxwellian distribution.

6. Averaging the collision strengths $\Omega_{j i}\left(E_{i}\right)$ over the regularlyspaced grid in $\log \left(E_{i} /\right.$ Ryd $)$ with a step of 0.01 gives the best approximation of the resulting $\Upsilon_{i j}(T, \kappa)$. The error for the strongest transitions is typically very small, less than $0.5 \%$. However, for weak transitions, low $\kappa \approx 2$, and ions formed at transition-region temperatures the error can reach $\approx 20-30 \%$. There are two reasons for this: the shift of the relative ion abundance to $\operatorname{low} \log (T / \mathrm{K})$ for low $\kappa$, as well as the strongly decreasing $\Omega_{j i}\left(E_{i}\right)$ with $E_{i}$ for such weak transitions. In these cases, the $\Upsilon_{i j}(T, \kappa)$ calculated from the averaged $\left\langle\Omega_{j i}\right\rangle_{\Delta \log \left(E_{i} / \mathrm{Ryd}\right)=0.01}$ are dominated by the errors in the approximation near its first energy point. However, given that the first energy point always has a large uncertainty in the scattering calculations, we still consider the error of $\approx 20-30 \%$ for weak transitions an acceptable one. We recommend using this method to decrease the size of the $\Omega_{j i}\left(E_{i}\right)$ datasets.

We have investigated all strong lines throughout the wavelength range unrestricted by the constraints of a given instrument. We have found only a few diagnostic options for $\kappa$ using lines of a single ion. Therefore, the diagnostic options including lines from neighbouring ionization stages need to be investigated in the future. Direct modelling of the entire observed spectrum including a few lines sensitive to $\kappa$ would be the best possibility. However, this is beyond the scope of this paper. Increase of sensitivity to $\kappa$ is expected if lines from neighbouring ionization stages are included (Dzifčáková \& Kulinová 2010; Mackovjak et al. 2013) because of the changes in the ionization equilibrium (Dzifčáková \& Dudík 2013, Fig. 6 therein). The ionization equilibrium is, however, an additional source of uncertainty because of the individual ionization and recombination rates, as well as possible departures from the ionization equilibrium (e.g. Bradshaw et al. 2004; Bradshaw 2009; de Avillez \& Breitschwerdt 2012; Reale et al. 2012; Doyle et al. 2012, 2013; Olluri et al. 2013) at low electron densities.

The results presented here highlight the need for spectroscopic observations over a wide wavelength range, especially in the $170 \AA-370 \AA$ range, which include many strongly temperature-sensitive line pairs, such as the Fe IX $244.909 \AA / 171.073 \AA$ or $244.909 \AA / 188.497 \AA$, or analogous combinations from other ions investigated here. Such a large wavelength range is necessary to keep the best options for diagnosing $\kappa$ from EUV lines. The proposed LEMUR instrument (Teriaca et al. 2012) will, however, observe the $170 \AA-210 \AA$ wavelength range together with many other spectral windows longward of $482 \AA$. These will include the Fe XII 1242.01 $⿱$ line together with the strongest Fe XII EUV lines, which may lead to a successful diagnostics. Inclusion of the forbidden lines in the near-UV, visible, or the infrared parts of the spectrum could, in principle, be done by multi-instrument observations involving space-borne spectrometers together with eclipse observations or ground-based coronagraphs, such as the COMP-S instrument being installed at the Lomnicky Peak observatory. 
Finally, we stress the particular importance and necessity of high-quality intensity calibration. Given the sensitivities to departures from the Maxwellian distribution presented here, the $\approx 20 \%$ uncertainties may no longer be sufficient enough.

Acknowledgements. The authors thank Peter Cargill for stimulating discussions. J.D. acknowledges support from the Royal Society via the Newton Fellowships Programme. G.D.Z. and H.E.M. acknowledge STFC funding through the DAMTP astrophysics grant, as well as the University of Strathclyde UK APAP network grant ST/J000892/1. E.Dz. acknowledges Grant 209/12/1652 of the Grant Agency of the Czech Republic. The authors also acknowledge the support from the International Space Science Institute through its International Teams program. Hinode is a Japanese mission developed and launched by ISAS/JAXA, with NAOJ as domestic partner and NASA and STFC (UK) as international partners. It is operated by these agencies in cooperation with ESA and NSC (Norway). CHIANTI is a collaborative project involving the NRL (USA), the University of Cambridge (UK), and George Mason University (USA).

\section{References}

Badnell, N. R. 1997, J. Phys. B Atom. Mol. Phys., 30, 1

Badnell, N. R. 2011, Comput. Phys. Commun., 182, 1528

Binette, L., Matadamas, R., Hägele, G. F., et al. 2012, A\&A, 547, A29

Bradshaw, S. J. 2009, A\&A, 502, 409

Bradshaw, S. J., \& Raymond, J. 2013, Space Sci. Rev., 178, 271

Bradshaw, S. J., Del Zanna, G., \& Mason, H. E. 2004, A\&A, 425, 287

Bryans, P. 2006, On the spectral emission of non-Maxwellian plasmas, Ph.D. thesis (University of Strathclyde)

Burgess, A., \& Tully, J. A. 1992, A\&A, 254, 436

Collier, M. R. 2004, Adv. Space Res., 33, 2108

Collier, M. R., Hamilton, D. C., Gloeckler, G., Bochsler, P., \& Sheldon, R. B. 1996, Geophys. Res. Lett., 23, 1191

Culhane, J. L., Harra, L. K., James, A. M., et al. 2007, Sol. Phys., 243, 19

de Avillez, M. A., \& Breitschwerdt, D. 2012, ApJ, 756, L3

De Pontieu, B., Title, A. M., Lemen, J. R., et al. 2014, Sol. Phys., 289, 2733

Del Zanna, G. 2010, A\&A, 514, A41

Del Zanna, G. 2011a, A\&A, 533, A12

Del Zanna, G. 2011b, A\&A, 536, A59

Del Zanna, G. 2012, A\&A, 537, A38

Del Zanna, G. 2013, A\&A, 555, A47

Del Zanna, G., \& Mason, H. E. 2005, A\&A, 433, 731

Del Zanna, G., \& Storey, P. J. 2012, A\&A, 543, A144

Del Zanna, G., \& Storey, P. J. 2013, A\&A, 549, A42

Del Zanna, G., Berrington, K. A., \& Mason, H. E. 2004, A\&A, 422, 731

Del Zanna, G., Storey, P. J., \& Mason, H. E. 2010, A\&A, 514, A40

Del Zanna, G., Storey, P. J., Badnell, N. R., \& Mason, H. E. 2012a, A\&A, 541, A90

Del Zanna, G., Storey, P. J., Badnell, N. R., \& Mason, H. E. 2012b, A\&A, 543, A139

Del Zanna, G., Storey, P. J., Badnell, N. R., \& Mason, H. E. 2014, A\&A, 565, A77

Dere, K. P. 2007, A\&A, 466, 771

Dere, K. P., Landi, E., Mason, H. E., Monsignori Fossi, B. C., \& Young, P. R. 1997, A\&AS, 125, 149

Dopita, M. A., Sutherland, R. S., Nicholls, D. C., Kewley, L. J., \& Vogt, F. P. A. 2013, ApJS, 208, 10

Doyle, J. G., Giunta, A., Singh, A., et al. 2012, Sol. Phys., 280, 111

Doyle, J. G., Giunta, A., Madjarska, M. S., et al. 2013, A\&A, 557, L9

Dzifčáková, E. 1992, Sol. Phys., 140, 247

Dzifčáková, E. 2002, Sol. Phys., 208, 91

Dzifčáková, E., \& Dudík, J. 2013, ApJS, 206, 6

Dzifčáková, E., \& Kulinová, A. 2010, Sol. Phys., 263, 25

Dzifčáková, E., \& Kulinová, A. 2011, A\&A, 531, A122
Dzif̌cáková, E., \& Mason, H. E. 2008, Sol. Phys., 247, 301

Dzifčáková, E., Homola, M., \& Dudík, J. 2011, A\&A, 531, A111

Feldman, U., Landi, E., \& Doschek, G. A. 2007, ApJ, 660, 1674

Flower, D. R., \& Pineau des Forets, G. 1973, A\&A, 24, 181

Habbal, S. R., Morgan, H., Druckmüller, M., et al. 2013, Sol. Phys., 285, 9

Hannah, I. G., Hudson, H. S., Hurford, G. J., \& Lin, R. P. 2010, ApJ, 724, 487

Kašparová, J., \& Karlický, M. 2009, A\&A, 497, L13

Landi, E., \& Young, P. R. 2010, ApJ, 714, 636

Landi, E., Del Zanna, G., Young, P. R., et al. 2006, ApJS, 162, 261

Landi, E., Del Zanna, G., Young, P. R., Dere, K. P., \& Mason, H. E. 2012, ApJ, 744, 99

Landi, E., Young, P. R., Dere, K. P., Del Zanna, G., \& Mason, H. E. 2013, ApJ, 763,86

Le Chat, G., Issautier, K., Meyer-Vernet, N., \& Hoang, S. 2011, Sol. Phys., 271, 141

Liang, G. Y., Whiteford, A. D., \& Badnell, N. R. 2009, A\&A, 500, 1263

Liang, G. Y., Badnell, N. R., Crespo López-Urrutia, J. R., et al. 2010, ApJS, 190, 322

Liang, G. Y., Badnell, N. R., \& Zhao, G. 2012, A\&A, 547, A87

Lin, R. P., Dennis, B. R., Hurford, G. J., et al. 2002, Sol. Phys., 210, 3

Livadiotis, G., \& McComas, D. J. 2009, J. Geophys. Res., 114, A11105

Livadiotis, G., \& McComas, D. J. 2010, ApJ, 714, 971

Livadiotis, G., \& McComas, D. J. 2011, ApJ, 741, 88

Livadiotis, G., \& McComas, D. J. 2013, Space Sci. Rev., 175, 183

Mackovjak, Š., Dzifčáková, E., \& Dudík, J. 2013, Sol. Phys., 282, 263

Maksimovic, M., Pierrard, V., \& Lemaire, J. F. 1997a, A\&A, 324, 725

Maksimovic, M., Pierrard, V., \& Riley, P. 1997b, Geophys. Res. Lett., 24, 1151

Mason, H. E., \& Monsignori Fossi, B. C. 1994, A\&ARv, 6, 123

Nicholls, D. C., Dopita, M. A., \& Sutherland, R. S. 2012, ApJ, 752, 148

Nicholls, D. C., Dopita, M. A., Sutherland, R. S., Kewley, L. J., \& Palay, E. 2013, ApJS, 207, 21

O’Dwyer, B., Del Zanna, G., Badnell, N. R., Mason, H. E., \& Storey, P. J. 2012, A\&A, 537, A22

Oka, M., Ishikawa, S., Saint-Hilaire, P., Krucker, S., \& Lin, R. P. 2013, ApJ, 764, 6

Olluri, K., Gudiksen, B. V., \& Hansteen, V. H. 2013, ApJ, 767, 43

Owocki, S. P., \& Scudder, J. D. 1983, ApJ, 270, 758

Phillips, K. J. H., Feldman, U., \& Landi, E. 2008, Ultraviolet and X-ray Spectroscopy of the Solar Atmosphere (Cambridge University Press)

Pinfield, D. J., Keenan, F. P., Mathioudakis, M., et al. 1999, ApJ, 527, 1000

Reale, F., Landi, E., \& Orlando, S. 2012, ApJ, 746, 18

Schwartz, P., Rybák, J., Kučera, A., et al. 2012, Contributions of the Astronomical Observatory Skalnate Pleso, 42, 135

Schwartz, P., Ambroz, J., Gömöry, P., et al. 2014, in IAU Symp., 300, 521

Scudder, J. D., \& Karimabadi, H. 2013, ApJ, 770, 26

Seaton, M. J., 1953, Roy. Soc. Lond. Proc. Ser. A, 218, 400

Singh, J., Sakurai, T., Ichimoto, K., \& Takeda, A. 2002, PASJ, 54, 807

Storey, P. J., \& Sochi, T. 2014, MNRAS, 440, 2581

Storey, P. J., Zeippen, C. J., \& Le Dourneuf, M. 2002, A\&A, 394, 753

Storey, P. J., Sochi, T., \& Badnell, N. R. 2014, MNRAS, 441, 3028

Teriaca, L., Andretta, V., Auchère, F., et al. 2012, Exp. Astron., 34, 273

Tomczyk, S., \& McIntosh, S. W. 2009, ApJ, 697, 1384

Tomczyk, S., McIntosh, S. W., Keil, S. L., et al. 2007, Science, 317, 1192

Tsallis, C. 1988, J. Stat. Phys., 52, 479

Tsallis, C. 2009, Introduction to Nonextensive Statistical Mechanics (New York: Springer)

Wang, T., Thomas, R. J., Brosius, J. W., et al. 2011, ApJS, 197, 32

Wannawichian, S., Ruffolo, D., \& Kartavykh, Y. Y. 2003, ApJS, 146, 443

Watanabe, T., Hara, H., Yamamoto, N., et al. 2009, ApJ, 692, 1294

Wiik, J. E., Schmieder, B., \& Noens, J. C. 1994, Sol. Phys., 149, 51

Wilhelm, K., Curdt, W., Marsch, E., et al. 1995, Sol. Phys., 162, 189

Young, P. R. 2009, ApJ, 691, L77

Young, P. R., Watanabe, T., Hara, H., \& Mariska, J. T. 2009, A\&A, 495, 587

Zouganelis, I. 2008, J. Geophys. Res., 113, A08111 


\section{Appendix A: Collision strength approximations and their accuracy}

Because of the large number of transitions in the $\mathrm{Fe}$ ions, the $\Omega_{j i}$ datafiles are several tens of GiB large. This hampers effective storage, handling, and publishing of such large datasets. Furthermore, calculations for some ions do not contain $\approx 10^{4} E_{i}^{(q)}$ points, but up to one order of magnitude more. An example is the O IV calculations done by Liang et al. (2012), which contain $\approx 1.87 \times 10^{5}$ points. We note that this ion was partially investigated already by Dudík et al. (2014).

In an effort to overcome the problems posed by the large data volume of the collison strength calculations, we tested whether coarser $E_{i}^{(q)}$ grids could be sufficient. To do so, we devised and tested several simple methods of averaging over the $E_{i}$ points:

1. Averaging over uniformly spaced grid of $E_{i}^{(q)}$ points, with $\Delta E_{i}^{(q)}=1 \mathrm{Ryd}$

2. Averaging over uniformly spaced grid of $E_{i}^{(q)}$ points, with $\Delta E_{i}^{(q)}=0.1 \mathrm{Ryd}$

3. Averaging over uniformly spaced grid in $\log \left(E_{i}^{(q)}\right)$, with $\Delta \log \left(E_{i}^{(q)} / \mathrm{Ryd}\right)=0.05$

4. Averaging over uniformly spaced grid in $\log \left(E_{i}^{(q)}\right)$, with $\Delta \log \left(E_{i}^{(q)} / \mathrm{Ryd}\right)=0.01$.

An example of the $\left\langle\Omega_{j i}\right\rangle$ obtained by these methods is shown in Fig. A.1 (top row) for O IV and Fe XI. The O IV is used here since its $\Omega_{i j}\left(E_{i}\right)$ has more than one order of magnitude more energy points than the Fe IX-Fe XIII ions studied in this paper. We note that in Fig. A.1, the $\mathrm{J}_{j i}(T, \kappa)$ are plotted for $\kappa=2$ instead of $\Upsilon_{j i}(T, \kappa)$ because of the $\exp \left(\Delta E_{i j} / k_{\mathrm{B}} T\right)$ factor present in Eq. (13) reaches very large values for $\operatorname{low} \log (T / \mathrm{K})$. The level of reduction of $E_{i}^{(q)}$ points depends strongly on the number of the original points, as well as the original interval covered by the $E_{i}^{(q)}$ points. Typically, the reduction is about an order of magnitude in case of the less coarse Methods 2 and 4, but can be much larger, e.g. a factor of $\approx 5 \times 10^{3}$ for O IV and Method 1 .
Upon calculation of $\Upsilon_{i j}(T, \kappa)$ and $\boldsymbol{J}_{j i}(T, \kappa)$, we found that Method 1 fails dramatically, since the $\Upsilon_{i j}\left(\left\langle\Omega_{j i}\right\rangle_{\Delta E_{i}=1 \text { Ryd }}\right)$ do not match the $\Upsilon_{i j}\left(\Omega_{j i}\right)$ calculated using the original data. Method 4 gives the best results in terms of most closely matching the $\Upsilon_{i j}(T, \kappa)$ and $\boldsymbol{J}_{j i}(T, \kappa)$ (see Fig. A.1) calculated using original, non-averaged $\Omega_{j i}$ data. Differences can arise at $\operatorname{low} \log (T / \mathrm{K})$ because at $\operatorname{low} \log (T / \mathrm{K})$, the $\Upsilon_{i j}(T, \kappa)$ and $\mathrm{J}_{j i}(T, \kappa)$ are dominated by the contribution from the energies near the excitation threshold at $E_{i}=\Delta E_{i j}$. The averaging of $\Omega_{j i}$ cannot be performed in the energy interval of the coarse $E_{i}$ grid containing the excitation threshold. As a result, inaccurate extrapolation of the first point of the $\left\langle\Omega_{j i}\right\rangle$ to the energy $\Delta E_{i j}$ causes departure of the $\Upsilon_{i j}\left(\left\langle\Omega_{j i}\right\rangle_{\Delta \log \left(E_{i} / \text { Ryd }\right)=0.01}\right)$ from the $\Upsilon_{i j}\left(\Omega_{j i}\right)$ calculated using the original $\Omega_{j i}$ data.

For Method 4, the relative error $R$, defined as

$R=\frac{\Upsilon_{i j}\left(\left\langle\Omega_{j i}\right\rangle_{\Delta \log \left(E_{i} / \mathrm{Ryd}\right)=0.01}\right)}{\Upsilon_{i j}\left(\Omega_{j i}\right)}-1$

is typically only a few per cent (see Fig. A.2). In this figure, we plot the relative error at temperatures corresponding to the temperature of the maximum relative ion abundance (Dzifčáková \& Dudík 2013). For the transitions having high rates (i.e. $\Upsilon_{i j}$ values), the relative error is very small, less than $0.5 \%$ (Fig. A.2). The relative error is larger for weaker transitions with lower $\Upsilon_{i j}$ values. For such transitions, the error is typically a few per cent. However, it can reach $\approx 20-30 \%$ for $\kappa=2$ and weak (unobservable) transitions in O IV.

The O IV and $\kappa=2$ is an extreme case for two reasons. First, the collision strength $\Omega_{j i}$ for the weak transitions are steeply decreasing with $E_{i}$, and thus the values of $\Upsilon_{i j}\left(\left\langle\Omega_{j i}\right\rangle_{\Delta \log \left(E_{i} / \mathrm{Ryd}\right)=0.01}\right)$ are dominated by the first energy point. Second, the maximum of the relative ion abundance of the O IV ion is, for $\kappa$-distributions, shifted to lower $\log (T / K)$ than for the Maxwellian distribution (Dzifčáková \& Dudík 2013; Dudík et al. 2014), increasing the error of the calculation. We consider even this value of $R=\approx 20-30 \%$ acceptable given the uncertainties in the atomic data calculations, particularly at the excitation threshold. We also note that the strongest transitions have small relative errors of only a few percentage points (Fig. A.2, bottom left). 
J. Dudík et al.: Signatures of the non-Maxwellian $\kappa$-distributions in optically thin line spectra
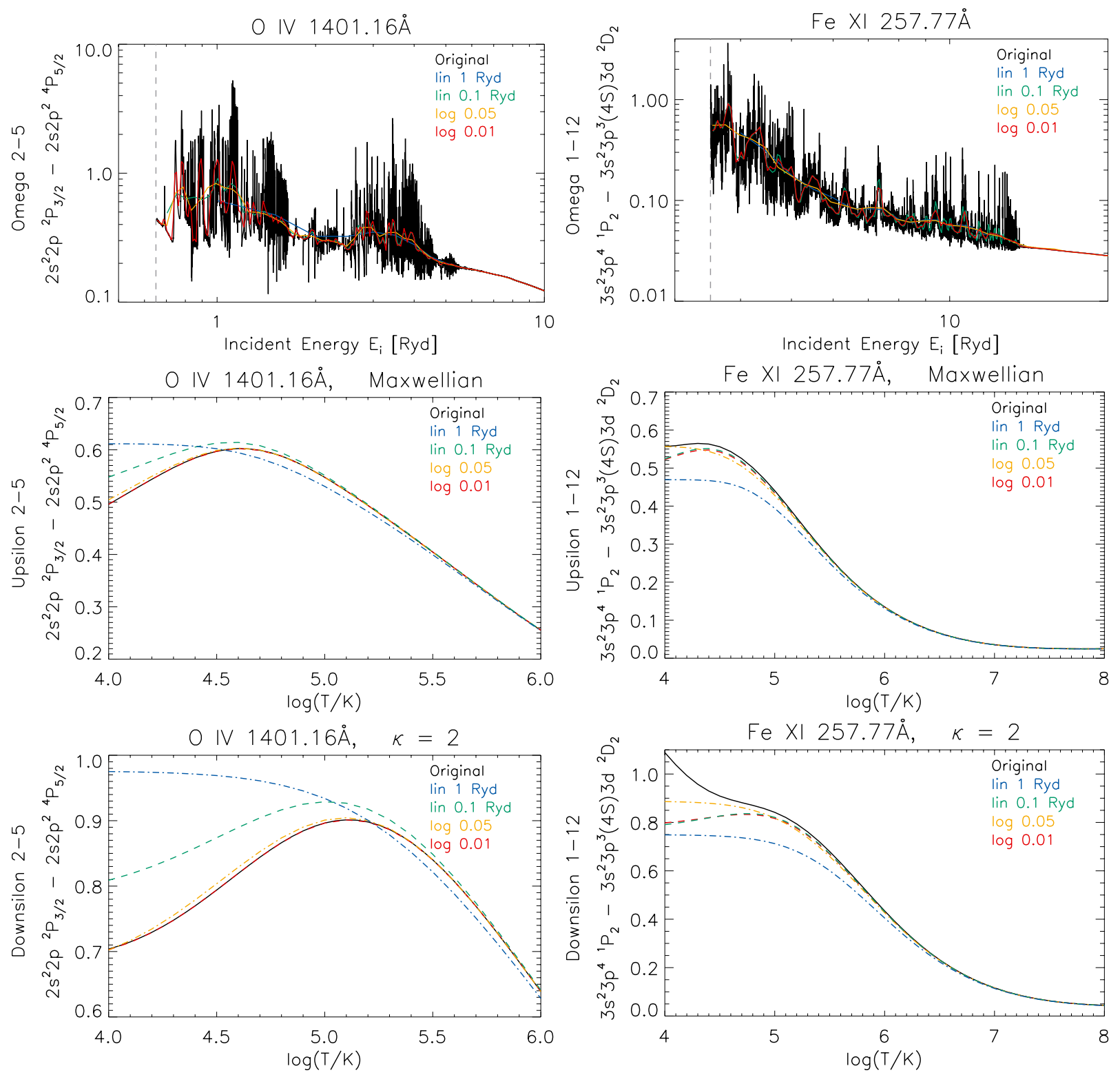

Fig. A.1. Examples of the collision strengths. Top: original collision strengths $\Omega_{i j}\left(E_{i}\right)$ (black) for the $1404.16 \AA$ transition in O IV (left) and the $257.77 \AA$ A transition in Fe XI (right). The colored lines correspond to the four averaging methods (Appendix A). Middle: Maxwellian $\Upsilon_{j i}$ for these transitions together with the $\Upsilon$ s obtained using the averaged $\left\langle\Omega_{i j}\left(E_{i}\right)\right\rangle$. Bottom: the same as the middle panel for $\kappa=2$. 

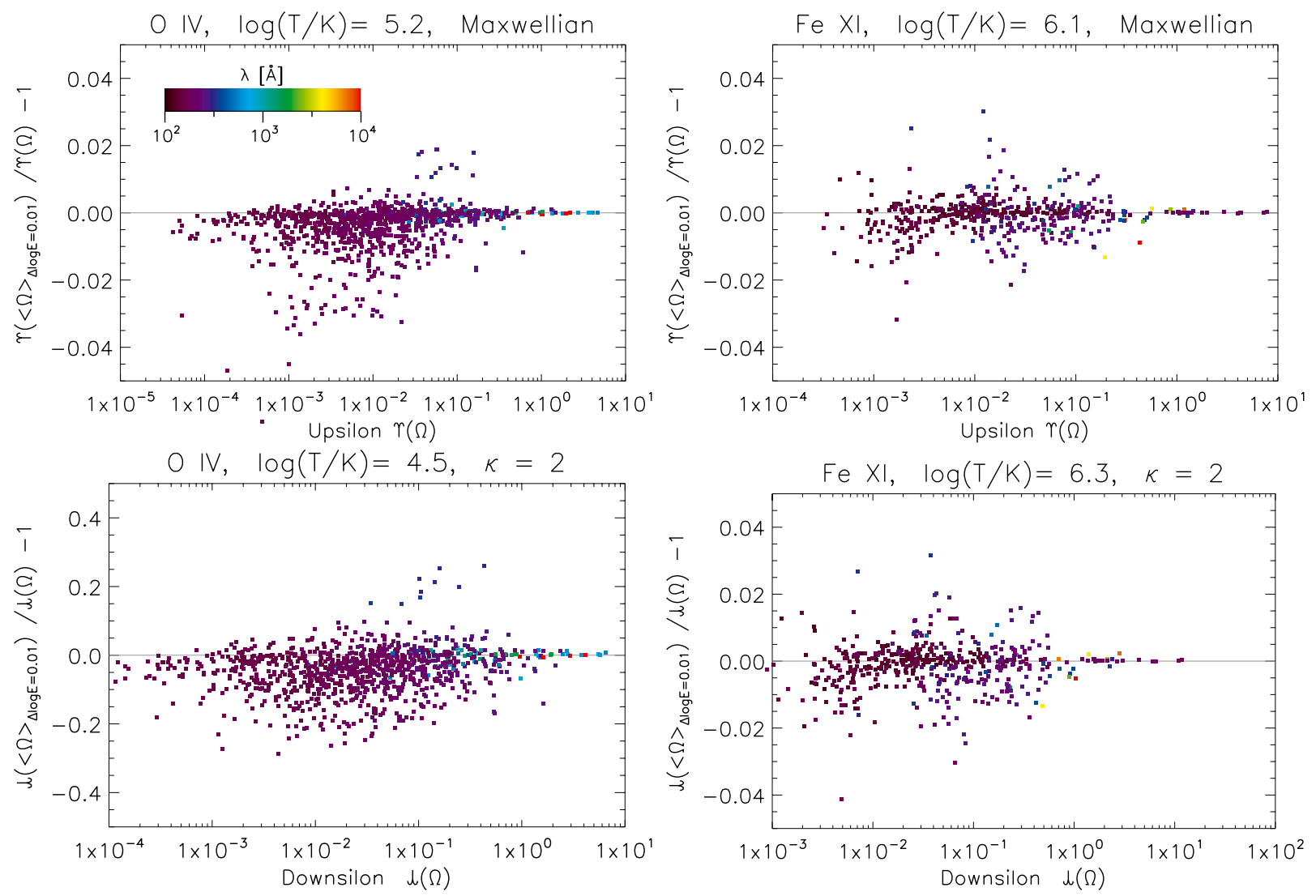

Fig. A.2. Relative error of the distribution-averaged collision strengths for O IV and Fe XI, produced using Method 4 (Appendix A), is shown for two extreme cases: Maxwellian distribution (top) and $k=2$ (bottom). The temperature corresponds to the maximum of the relative ion abundance. 


\section{Appendix B: Selected transitions}

A list of the spectral lines selected by the procedure described in Sect. 5.1 are listed in Tables B.1-B.5 together with their selfblends. Wavelengths of the primary (strongest) transition within a selfblend are given together with the energy levels involved. Selfblending transitions are indicated together with their level numbers. Most of the selected spectral lines do not contain any selflblending transitions.

Table B.1. Fe IX lines selected with the procedure described in Sect. 5.1.

\begin{tabular}{|c|c|c|c|c|c|c|c|}
\hline$\lambda[\AA]$ & Levels & Level description & $I(\mathrm{Maxw})$ & $I(\kappa=5)$ & $I(\kappa=2)$ & Selfblends & Notes \\
\hline 157.764 & $6-121$ & $3 s^{2} 3 p^{5} 3 d^{3} F_{3}-3 s^{2} 3 p^{4} 3 d^{2}{ }^{3} F_{3}$ & 0.0158 & 0.0098 & 0.0021 & & \\
\hline 168.080 & $5-118$ & $3 s^{2} 3 p^{5} 3 d^{3} F_{4}-3 s^{2} 3 p^{4} 3 d^{2}{ }^{3} F_{4}$ & 0.0362 & 0.0223 & 0.0047 & $\begin{array}{l}169.010(3-117) \\
169.048(8-131)\end{array}$ & $\begin{array}{l}\text { th } \\
\text { th }\end{array}$ \\
\hline 159.949 & $12-133$ & $3 s^{2} 3 p^{5} 3 d^{1} F_{3}-3 s^{2} 3 p^{4} 3 d^{2}{ }^{1} F_{3}$ & 0.0047 & 0.0030 & 0.0008 & $159.986(3-112)$ & \\
\hline 160.379 & $4-115$ & $3 s^{2} 3 p^{5} 3 d^{3} P_{2}-3 s^{2} 3 p^{4} 3 d^{2}{ }^{3} P_{2}$ & 0.0043 & 0.0029 & 0.0008 & & \\
\hline 162.945 & $8-124$ & $3 s^{2} 3 p^{5} 3 d^{3} D_{3}-3 s^{2} 3 p^{4} 3 d^{2}{ }^{3} D_{3}$ & 0.0089 & 0.0056 & 0.0013 & $162.901(9-125)$ & \\
\hline 171.073 & $1-13$ & $3 s^{2} 3 p^{6}{ }^{1} S_{0}-3 s^{2} 3 p^{5} 3 d^{1} P_{1}$ & 1.0000 & 0.7430 & 0.3092 & & \\
\hline 177.592 & $6-111$ & $3 s^{2} 3 p^{5} 3 d^{3} F_{3}-3 s^{2} 3 p^{4} 3 d^{2}{ }^{3} D_{2}$ & 0.0047 & 0.0031 & 0.0008 & & \\
\hline 176.930 & $12-113$ & $3 s^{2} 3 p^{5} 3 d^{1} F_{3}-3 s^{2} 3 p^{4} 3 d^{2}{ }^{1} G_{4}$ & 0.0188 & 0.0117 & 0.0026 & & \\
\hline 176.945 & $5-110$ & $3 s^{2} 3 p^{5} 3 d^{3} F_{4}-3 s^{2} 3 p^{4} 3 d^{2}{ }^{3} D_{3}$ & 0.0427 & 0.0262 & 0.0054 & $\begin{array}{l}165.876(6-113) \\
165.880(8-118) \\
165.908(12-123)\end{array}$ & $\begin{array}{l}\text { bl Fe VII } \\
\text { th } \\
\text { th }\end{array}$ \\
\hline 183.851 & $9-99$ & $3 s^{2} 3 p^{5} 3 d^{1} D_{2}-3 s^{2} 3 p^{4} 3 d^{2}{ }^{1} F_{3}$ & 0.0055 & 0.0035 & 0.0008 & $\begin{array}{l}183.877(12-105) \\
183.898(5-89)\end{array}$ & bl Fe VII \\
\hline 188.497 & $5-96$ & $3 s^{2} 3 p^{5} 3 d^{3} F_{4}-3 s^{2} 3 p^{4} 3 d^{2}{ }^{3} G_{5}$ & 0.0500 & 0.0307 & 0.0064 & & \\
\hline 189.941 & $6-95$ & $3 s^{2} 3 p^{5} 3 d^{3} F_{3}-3 s^{2} 3 p^{4} 3 d^{2}{ }^{3} G_{4}$ & 0.0299 & 0.0187 & 0.0041 & & \\
\hline 191.216 & $7-97$ & $3 s^{2} 3 p^{5} 3 d^{3} F_{2}-3 s^{2} 3 p^{4} 3 d^{2}{ }^{3} G_{3}$ & 0.0124 & 0.0079 & 0.0018 & & \\
\hline 194.784 & $4-85$ & $3 s^{2} 3 p^{5} 3 d_{p}^{3} 2-3 s^{2} 3 p^{4} 3 d^{2}{ }^{3} D_{3}$ & 0.0082 & 0.0052 & 0.0013 & & \\
\hline 197.862 & $13-148$ & $3 s^{2} 3 p^{5} 3 d^{1} P_{1}-3 s^{2} 3 p^{5} 4 p{ }^{1} S_{0}$ & 0.0233 & 0.0174 & 0.0072 & & \\
\hline 217.101 & $1-10$ & $3 s^{2} 3 p^{6}{ }^{1} S_{0}-3 s^{2} 3 p^{5} 3 d^{3} D_{1}$ & 0.0566 & 0.0439 & 0.0202 & & \\
\hline 218.937 & $1-9$ & $3 s^{2} 3 p^{6}{ }^{1} S_{0}-3 s^{2} 3 p^{5} 3 d^{1} D_{2}$ & 0.0308 & 0.0233 & 0.0100 & & \\
\hline 230.511 & $1-7$ & $3 s^{2} 3 p^{6}{ }^{1} S_{0}-3 s^{2} 3 p^{5} 3 d^{3} F_{2}$ & 0.0096 & 0.0071 & 0.0028 & $\begin{array}{l}230.475(11-55) \\
230.479(10-52)\end{array}$ & \\
\hline 241.739 & $1-4$ & $3 s^{2} 3 p^{6}{ }^{1} S_{0}-3 s^{2} 3 p^{5} 3 d^{3} P_{2}$ & 0.1849 & 0.1386 & 0.0590 & & \\
\hline 244.909 & $1-3$ & $3 s^{2} 3 p^{6}{ }^{1} S_{0}-3 s^{2} 3 p^{5} 3 d^{3} P_{1}$ & 0.0998 & 0.0773 & 0.0369 & & \\
\hline 317.193 & $4-16$ & $3 s^{2} 3 p^{5} 3 d^{3} P_{2}-3 s 3 p^{6} 3 d^{3} D_{3}$ & 0.0121 & 0.0083 & 0.0024 & & \\
\hline 329.897 & $5-16$ & $3 s^{2} 3 p^{5} 3 d^{3} F_{4}-3 s 3 p^{6} 3 d^{3} D_{3}$ & 0.0153 & 0.0105 & 0.0031 & & \\
\hline 352.060 & $12-17$ & $3 s^{2} 3 p^{5} 3 d^{1} F_{3}-3 s 3 p^{6} 3 d^{1} D_{2}$ & 0.0168 & 0.0122 & 0.0048 & & \\
\hline 2043.01 & $4-11$ & $3 s^{2} 3 p^{5} 3 d^{3} P_{2}-3 s^{2} 3 p^{5} 3 d^{3} D_{2}$ & 0.0497 & 0.0375 & 0.0158 & & \\
\hline 2384.19 & $4-8$ & $3 s^{2} 3 p^{5} 3 d_{p}^{3} 2-3 s^{2} 3 p^{5} 3 d^{3} D_{3}$ & 0.0432 & 0.0318 & 0.0124 & & \\
\hline 2498.84 & $5-12$ & $3 s^{2} 3 p^{5} 3 d^{3} F_{4}-3 s^{2} 3 p^{5} 3 d^{1} F_{3}$ & 0.0849 & 0.0649 & 0.0282 & & \\
\hline 3644.08 & $6-9$ & $3 s^{2} 3 p^{5} 3 d^{3} F_{3}-3 s^{2} 3 p^{5} 3 d^{1} D_{2}$ & 0.0240 & 0.0182 & 0.0078 & & \\
\hline 3802.10 & $6-8$ & $3 s^{2} 3 p^{5} 3 d^{3} F_{3}-3 s^{2} 3 p^{5} 3 d^{3} D_{3}$ & 0.0264 & 0.0194 & 0.0075 & & \\
\hline 22183.0 & $6-7$ & $3 s^{2} 3 p^{5} 3 d^{3} F_{3}-3 s^{2} 3 p^{5} 3 d^{3} F_{2}$ & 0.0149 & 0.0111 & 0.0044 & & \\
\hline 28563.0 & $5-6$ & $3 s^{2} 3 p^{5} 3 d^{3} F_{4}-3 s^{2} 3 p^{5} 3 d^{3} F_{3}$ & 0.0101 & 0.0073 & 0.0027 & & \\
\hline
\end{tabular}

Notes. Strongest transitions are listed together with the wavelengths of their selfblends. Intensities relative to the strongest line are listed for the Maxwellian distribution, $k=5$ and 2, for temperature and density corresponding to Fig. 3. Blended transitions are indicated by "bl"; "th" denotes that the wavelength is theoretical only; "w" denotes a line that is observed to be weak. 
Table B.2. Same as Table B.1, but for Fe X.

\begin{tabular}{|c|c|c|c|c|c|c|c|}
\hline$\lambda[\AA]$ & Levels & Level description & $I(\mathrm{Maxw})$ & $I(\kappa=5)$ & $I(\kappa=2)$ & Selfblends & Notes \\
\hline 174.531 & $1-30$ & $3 s^{2} 3 p^{5}{ }^{2} P_{3 / 2}-3 s^{2} 3 p^{4} 3 d^{2} D_{5 / 2}$ & 1.0000 & 0.8535 & 0.3956 & $174.482(10-145)$ & \\
\hline 175.263 & $2-31$ & $3 s^{2} 3 p^{5}{ }^{2} P_{1 / 2}-3 s^{2} 3 p^{4} 3 d^{2} D_{3 / 2}$ & 0.1115 & 0.1042 & 0.0670 & $\begin{array}{l}175.223(18-155) \\
175.280(18-154)\end{array}$ & \\
\hline 175.475 & $1-29$ & $3 s^{2} 3 p^{5}{ }^{2} P_{3 / 2}-3 s^{2} 3 p^{4} 3 d^{2} P_{1 / 2}$ & 0.0308 & 0.0273 & 0.0147 & $175.487(17-153)$ & \\
\hline 177.240 & $1-28$ & $3 s^{2} 3 p^{5}{ }^{2} \mathrm{P}_{3 / 2}-3 s^{2} 3 p^{4} 3 d^{2} \mathrm{P}_{3 / 2}$ & 0.5731 & 0.4905 & 0.2293 & & \\
\hline 180.441 & $2-29$ & $3 s^{2} 3 p^{5}{ }^{2} \mathrm{P}_{1 / 2}-3 s^{2} 3 p^{4} 3 d^{2} \mathrm{P}_{1 / 2}$ & 0.0798 & 0.0707 & 0.0380 & & bl Fe XI \\
\hline 184.537 & $1-27$ & $3 s^{2} 3 p^{5}{ }^{2} \mathrm{P}_{3 / 2}-3 s^{2} 3 p^{4} 3 d^{2} S_{1 / 2}$ & 0.2291 & 0.1980 & 0.0958 & $184.509(20-144)$ & \\
\hline 190.037 & $2-27$ & $3 s^{2} 3 p^{5}{ }^{2} P_{1 / 2}-3 s^{2} 3 p^{4} 3 d^{2} S_{1 / 2}$ & 0.0777 & 0.0671 & 0.0325 & $\begin{array}{l}189.992(23-153) \\
189.996(19-131)\end{array}$ & bl Fe XII \\
\hline 193.715 & $1-26$ & $3 s^{2} 3 p^{5}{ }^{2} P_{3 / 2}-3 s^{2} 3 p^{4} 3 d^{2} D_{5 / 2}$ & 0.0308 & 0.0288 & 0.0168 & & \\
\hline 207.449 & $1-23$ & $3 s^{2} 3 p^{5}{ }^{2} \mathrm{P}_{3 / 2}-3 s^{2} 3 p^{4} 3 d^{2} F_{5 / 2}$ & 0.0359 & 0.0337 & 0.0202 & & \\
\hline 220.247 & $1-21$ & $3 s^{2} 3 p^{5}{ }^{2} \mathrm{P}_{3 / 2}-3 s^{2} 3 p^{4} 3 d^{2} F_{5 / 2}$ & 0.0512 & 0.0476 & 0.0287 & & \\
\hline 224.800 & $1-11$ & $3 s^{2} 3 p^{5}{ }^{2} \mathrm{P}_{3 / 2}-3 s^{2} 3 p^{4} 3 d^{2} P_{3 / 2}$ & 0.0327 & 0.0306 & 0.0185 & & \\
\hline 225.856 & $1-19$ & $3 s^{2} 3 p^{5}{ }^{2} P_{3 / 2}-3 s^{2} 3 p^{4} 3 d^{2} D_{5 / 2}$ & 0.0649 & 0.0602 & 0.0355 & & \\
\hline 226.998 & $1-17$ & $3 s^{2} 3 p^{5}{ }^{2} \mathrm{P}_{3 / 2}-3 s^{2} 3 p^{4} 3 d^{4} \mathrm{P}_{5 / 2}$ & 0.0451 & 0.0421 & 0.0250 & & \\
\hline 234.315 & $1-12$ & $3 s^{2} 3 p^{5}{ }^{2} \mathrm{P}_{3 / 2}-3 s^{2} 3 p^{4} 3 d^{4} F_{5 / 2}$ & 0.0475 & 0.0456 & 0.0297 & & bl He II \\
\hline 256.398 & $1-6$ & $3 s^{2} 3 p^{5}{ }^{2} P_{3 / 2}-3 s^{2} 3 p^{4} 3 d^{4} D_{3 / 2}$ & 0.0344 & 0.0336 & 0.0231 & & bl He II \\
\hline 257.263 & $1-5$ & $3 s^{2} 3 p^{5}{ }^{2} P_{3 / 2}-3 s^{2} 3 p^{4} 3 d^{4} D_{7 / 2}$ & 0.2397 & 0.2148 & 0.1110 & $\begin{array}{l}257.257(24-81) \\
257.259(1-4)\end{array}$ & \\
\hline 345.738 & $1-3$ & $3 s^{2} 3 p^{5}{ }^{2} \mathrm{P}_{3 / 2}-3 \mathrm{~s} 3 \mathrm{p}^{6}{ }^{2} S_{1 / 2}$ & 0.1476 & 0.1390 & 0.0886 & & \\
\hline 365.560 & $2-3$ & $3 s^{2} 3 p^{5}{ }^{2} \mathrm{P}_{1 / 2}-3 \mathrm{~s} 3 \mathrm{p}^{6}{ }^{2} S_{1 / 2}$ & 0.0645 & 0.0608 & 0.0387 & & \\
\hline 3454.95 & $5-8$ & $3 s^{2} 3 p^{4} 3 d^{4} D_{7 / 2}-3 s^{2} 3 p^{4} 3 d^{4} F_{9 / 2}$ & 0.0582 & 0.0488 & 0.0191 & & \\
\hline 6376.29 & $1-2$ & $3 s^{2} 3 p^{5}{ }^{2} \mathrm{P}_{3 / 2}-3 s^{2} 3 p^{5}{ }^{2} \mathrm{P}_{1 / 2}$ & 0.6373 & 0.6969 & 0.8182 & & \\
\hline
\end{tabular}


Table B.3. Same as Table B.1, but for Fe XI.

\begin{tabular}{|c|c|c|c|c|c|c|c|}
\hline$\lambda[\AA]$ & Levels & Level description & $I(\mathrm{Maxw})$ & $I(\kappa=5)$ & $I(\kappa=2)$ & Selfblends & Notes \\
\hline 178.058 & $1-43$ & $3 s^{2} 3 p^{4}{ }^{3} P_{2}-3 s^{2} 3 p^{3} 3 d^{3} D_{2}$ & 0.0496 & 0.0416 & 0.0202 & & \\
\hline 179.758 & $4-46$ & $3 s^{2} 3 p^{4}{ }^{1} D_{2}-3 s^{2} 3 p^{3} 3 d^{1} F_{3}$ & 0.0497 & 0.0444 & 0.0257 & $\begin{array}{l}179.734(12-140) \\
179.772(15-158) \\
179.792(13-141) \\
179.820(18-164)\end{array}$ & \\
\hline 180.401 & $1-42$ & $3 s^{2} 3 p^{4}{ }^{3} P_{2}-3 s^{2} 3 p^{3} 3 d^{3} D_{3}$ & 1.0000 & 0.8171 & 0.3574 & $180.392(8-100)$ & bl FeX \\
\hline 180.594 & $2-44$ & $3 s^{2} 3 p^{4}{ }^{3} P_{1}-3 s^{2} 3 p^{3} 3 d^{3} D_{1}$ & 0.0417 & 0.0361 & 0.0193 & $\begin{array}{l}180.554(17-160) \\
180.643(21-167)\end{array}$ & \\
\hline 181.130 & $3-44$ & $3 s^{2} 3 p^{4}{ }^{3} P_{0}-3 s^{2} 3 p^{3} 3 d^{3} D_{1}$ & 0.0581 & 0.0503 & 0.0270 & & \\
\hline 182.167 & $2-43$ & $3 s^{2} 3 p^{4}{ }^{3} P_{1}-3 s^{2} 3 p^{3} 3 d^{3} D_{2}$ & 0.1810 & 0.1518 & 0.0739 & & \\
\hline 184.410 & $5-48$ & $3 s^{2} 3 p^{4}{ }^{1} S_{0}-3 s^{2} 3 p^{3} 3 d^{1} P_{1}$ & 0.0046 & 0.0041 & 0.0022 & $184.446(36-216)$ & \\
\hline 184.793 & $4-45$ & $3 s^{2} 3 p^{4}{ }^{1} D_{2}-3 s^{2} 3 p^{3} 3 d^{1} D_{2}$ & 0.0311 & 0.0278 & 0.0161 & $184.805(32-193)$ & \\
\hline 188.216 & $1-38$ & $3 s^{2} 3 p^{4}{ }^{3} P_{2}-3 s^{2} 3 p^{3} 3 d^{3} P_{2}$ & 0.5096 & 0.4196 & 0.1884 & & \\
\hline 188.299 & $1-37$ & $3 s^{2} 3 p^{4}{ }^{3} P_{2}-3 s^{2} 3 p^{3} 3 d^{1} P_{1}$ & 0.2901 & 0.2383 & 0.1061 & & \\
\hline 188.997 & $2-40$ & $3 s^{2} 3 p^{4}{ }^{3} P_{1}-3 s^{2} 3 p^{3} 3 d^{3} P_{0}$ & 0.0271 & 0.0238 & 0.0134 & & \\
\hline 189.123 & $2-41$ & $3 s^{2} 3 p^{4}{ }^{3} P_{1}-3 s^{2} 3 p^{3} 3 d^{3} P_{1}$ & 0.0239 & 0.0207 & 0.0110 & & \\
\hline 189.711 & $3-41$ & $3 s^{2} 3 p^{4}{ }^{3} P_{0}-3 s^{2} 3 p^{3} 3 d^{3} P_{1}$ & 0.0201 & 0.0174 & 0.0093 & $189.736(30-184)$ & bl \\
\hline 190.382 & $1-36$ & $3 s^{2} 3 p^{4}{ }^{3} P_{2}-3 s^{2} 3 p^{3} 3 d^{1} F_{3}$ & 0.0221 & 0.0191 & 0.0099 & $190.390(7-85)$ & \\
\hline 192.021 & $2-39$ & $3 s^{2} 3 p^{4}{ }^{3} P_{1}-3 s^{2} 3 p^{3} 3 d^{3} S_{1}$ & 0.0219 & 0.0187 & 0.0097 & & bl \\
\hline 192.813 & $2-38$ & $3 s^{2} 3 p^{4}{ }^{3} P_{1}-3 s^{2} 3 p^{3} 3 d^{3} P_{2}$ & 0.1091 & 0.0898 & 0.0403 & & $\begin{array}{l}\text { bl Ca XVII } \\
\text { bl OV }\end{array}$ \\
\hline 192.900 & $2-37$ & $3 s^{2} 3 p^{4}{ }^{3} P_{1}-3 s^{2} 3 p^{3} 3 d^{1} P_{1}$ & 0.0605 & 0.0497 & 0.0221 & & $\begin{array}{l}\text { bl CaXVII } \\
\text { bl Ov }\end{array}$ \\
\hline 198.538 & $4-41$ & $3 s^{2} 3 p^{4}{ }^{1} D_{2}-3 s^{2} 3 p^{3} 3 d^{3} P_{1}$ & 0.0284 & 0.0246 & 0.0131 & & bl S VIII \\
\hline 201.112 & $1-35$ & $3 s^{2} 3 p^{4}{ }^{3} P_{2}-3 s^{2} 3 p^{3} 3 d^{3} D_{3}$ & 0.0304 & 0.0263 & 0.0138 & & bl Fe XIII \\
\hline 201.734 & $4-39$ & $3 s^{2} 3 p^{4}{ }^{1} D_{2}-3 s^{2} 3 p^{3} 3 d^{3} S_{1}$ & 0.0384 & 0.0328 & 0.0170 & & bl Fe XII \\
\hline 202.424 & $1-34$ & $3 s^{2} 3 p^{4}{ }^{3} P_{2}-3 s^{2} 3 p^{3} 3 d^{3} P_{2}$ & 0.0575 & 0.0482 & 0.0226 & $\begin{array}{l}202.388(13-117) \\
202.393(1-31) \\
202.461(14-118)\end{array}$ & \\
\hline 230.165 & $4-26$ & $3 s^{2} 3 p^{4}{ }^{1} D_{2}-3 s^{2} 3 p^{3} 3 d^{1} D_{2}$ & 0.0187 & 0.0171 & 0.0103 & & \\
\hline 234.730 & $1-20$ & $3 s^{2} 3 p^{4}{ }^{3} P_{2}-3 s^{2} 3 p^{3} 3 d^{3} F_{3}$ & 0.0240 & 0.0217 & 0.0130 & & \\
\hline 236.494 & $1-18$ & $3 s^{2} 3 p^{4}{ }^{3} P_{2}-3 s^{2} 3 p^{3} 3 d^{3} F_{2}$ & 0.0281 & 0.0258 & 0.0161 & & \\
\hline 239.787 & $4-22$ & $3 s^{2} 3 p^{4}{ }^{1} D_{2}-3 s^{2} 3 p^{3} 3 d^{3} G_{3}$ & 0.0316 & 0.0289 & 0.0177 & $239.780(4-22)$ & \\
\hline 240.717 & $1-16$ & $3 s^{2} 3 p^{4}{ }^{3} P_{2}-3 s^{2} 3 p^{3} 3 d^{3} D_{3}$ & 0.0810 & 0.0701 & 0.0373 & $240.757(9-75)$ & bl Fe XIII \\
\hline 242.215 & $1-15$ & $3 s^{2} 3 p^{4}{ }^{3} P_{2}-3 s^{2} 3 p^{3} 3 d^{3} D_{2}$ & 0.0354 & 0.0317 & 0.0185 & & \\
\hline 256.919 & $1-14$ & $3 s^{2} 3 p^{4}{ }^{3} P_{2}-3 s^{2} 3 p^{3} 3 d^{5} D_{4}$ & 0.0796 & 0.0695 & 0.0351 & & bl Fe XII \\
\hline 257.554 & $1-13$ & $3 s^{2} 3 p^{4}{ }^{3} P_{2}-3 s^{2} 3 p^{3} 3 d^{5} D_{3}$ & 0.0940 & 0.0862 & 0.0528 & $\begin{array}{l}257.538(22-96) \\
257.547(4-20) \\
257.558(42-147)\end{array}$ & \\
\hline 257.772 & $1-12$ & $3 s^{2} 3 p^{4}{ }^{3} P_{2}-3 s^{2} 3 p^{3} 3 d^{5} D_{2}$ & 0.0365 & 0.0341 & 0.0220 & $257.725(23-97)$ & \\
\hline 264.772 & $4-16$ & $3 s^{2} 3 p^{4}{ }^{1} D_{2}-3 s^{2} 3 p^{3} 3 d^{3} D_{3}$ & 0.0309 & 0.0267 & 0.0142 & & \\
\hline 308.544 & $4-9$ & $3 \mathrm{~s}^{2} 3 \mathrm{p}^{4}{ }^{1} \mathrm{D}_{2}-3 \mathrm{~s} 3 \mathrm{p}^{5}{ }^{1} \mathrm{P}_{1}$ & 0.0384 & 0.0365 & 0.0251 & & \\
\hline 341.113 & $1-7$ & $3 s^{2} 3 p^{4}{ }^{3} \mathrm{P}_{2}-3 \mathrm{~s} 3 \mathrm{p}^{5}{ }^{3} \mathrm{P}_{1}$ & 0.0600 & 0.0542 & 0.0339 & & \\
\hline 349.046 & $2-8$ & $3 s^{2} 3 p^{4}{ }^{3} \mathrm{P}_{1}-3 s 3 p^{5}{ }^{3} \mathrm{P}_{0}$ & 0.0189 & 0.0183 & 0.0137 & & $\mathrm{bl} \mathrm{u}$ \\
\hline 352.670 & $1-6$ & $3 s^{2} 3 p^{4}{ }^{3} \mathrm{P}_{2}-3 s 3 p^{5}{ }^{3} \mathrm{P}_{2}$ & 0.2461 & 0.2188 & 0.1295 & $352.622(35-78)$ & \\
\hline 356.519 & $2-7$ & $3 s^{2} 3 p^{4}{ }^{3} \mathrm{P}_{1}-3 \mathrm{~s} 3 \mathrm{p}^{5}{ }^{3} \mathrm{P}_{1}$ & 0.0312 & 0.0282 & 0.0176 & & \\
\hline 358.613 & $3-7$ & $3 \mathrm{~s}^{2} 3 \mathrm{p}^{4}{ }^{3} \mathrm{P}_{0}-3 \mathrm{~s} 3 \mathrm{p}^{5}{ }^{3} \mathrm{P}_{1}$ & 0.0399 & 0.0361 & 0.0225 & & \\
\hline 369.163 & $2-6$ & $3 s^{2} 3 p^{4}{ }^{3} P_{1}-3 s 3 p^{5}{ }^{3} P_{2}$ & 0.0766 & 0.0681 & 0.0403 & & \\
\hline 2649.50 & $1-4$ & $3 s^{2} 3 p^{4}{ }^{3} P_{2}-3 s^{2} 3 p 64{ }^{1} D_{2}$ & 0.4325 & 0.4315 & 0.4003 & & \\
\hline 7894.03 & $1-2$ & $3 s^{2} 3 p^{4}{ }^{3} P_{2}-3 s^{2} 3 p^{4}{ }^{3} P_{1}$ & 0.6523 & 0.6240 & 0.5295 & & \\
\hline
\end{tabular}


Table B.4. Same as Table B.1, but for Fe XII.

\begin{tabular}{|c|c|c|c|c|c|c|c|}
\hline$\lambda[\AA]$ & Levels & Level description & $I(\mathrm{Maxw})$ & $I(\kappa=5)$ & $I(\kappa=2)$ & Selfblends & Notes \\
\hline 186.887 & $3-39$ & $3 s^{2} 3 p^{32} D_{5 / 2}-3 s^{2} 3 p^{2} 3 d^{2} F_{7 / 2}$ & 0.3499 & 0.2888 & 0.0953 & $\begin{array}{l}186.854(2-36) \\
186.931(22-135)\end{array}$ & \\
\hline 188.170 & $4-41$ & $3 s^{2} 3 p^{3}{ }^{2} P_{1 / 2}-3 s^{2} 3 p^{2} 3 d^{2} D_{3 / 2}$ & 0.0065 & 0.0057 & 0.0023 & $\begin{array}{l}188.114(3-38) \\
188.130(10-99)\end{array}$ & \\
\hline 190.467 & $5-41$ & $3 s^{2} 3 p^{3}{ }^{2} P_{3 / 2}-3 s^{2} 3 p^{2} 3 d^{2} D_{3 / 2}$ & 0.0038 & 0.0032 & 0.0011 & $190.487(17-130)$ & \\
\hline 191.049 & $5-40$ & $3 s^{2} 3 p^{3}{ }^{2} P_{3 / 2}-3 s^{2} 3 p^{2} 3 d^{2} D_{5 / 2}$ & 0.0208 & 0.0174 & 0.0059 & $\begin{array}{l}190.977(40-203) \\
191.017(6-78) \\
191.059(20-131) \\
191.074(33-178) \\
191.096(17-129)\end{array}$ & \\
\hline 192.394 & $1-30$ & $3 s^{2} 3 p^{3}{ }^{4} S_{3 / 2}-3 s^{2} 3 p^{2} 3 d^{4} P_{1 / 2}$ & 0.3143 & 0.2517 & 0.0783 & & \\
\hline 193.509 & $1-29$ & $3 s^{2} 3 p^{3}{ }^{4} S_{3 / 2}-3 s^{2} 3 p^{2} 3 d^{4} P_{3 / 2}$ & 0.6713 & 0.5380 & 0.1675 & $193.500(7-80)$ & \\
\hline 195.119 & $1-27$ & $3 s^{2} 3 p^{3}{ }^{4} S_{3 / 2}-3 s^{2} 3 p^{2} 3 d^{4} P_{5 / 2}$ & 1.0000 & 0.8026 & 0.2510 & $195.078(31-151)$ & sbl \\
\hline 195.179 & $2-33$ & $3 s^{2} 3 p^{3}{ }^{2} D_{3 / 2}-3 s^{2} 3 p^{2} 3 d^{2} D_{3 / 2}$ & 0.0373 & 0.0322 & 0.0122 & $195.221(13-115)$ & sbl \\
\hline 196.640 & $3-34$ & $3 s^{2} 3 p^{3}{ }^{2} D_{5 / 2}-3 s^{2} 3 p^{2} 3 d^{2} D_{5 / 2}$ & 0.0944 & 0.0776 & 0.0252 & $196.646(23-131)$ & \\
\hline 201.140 & $5-38$ & $3 s^{2} 3 p^{3}{ }^{2} \mathrm{P}_{3 / 2}-3 s^{2} 3 p^{2} 3 d^{2} \mathrm{P}_{3 / 2}$ & 0.0095 & 0.0080 & 0.0029 & $\begin{array}{l}201.133(11-104) \\
201.186(6-67)\end{array}$ & bl Fe XIII \\
\hline 201.740 & $4-35$ & $3 s^{2} 3 p^{3}{ }^{2} P_{1 / 2}-3 s^{2} 3 p^{2} 3 d^{2} P_{1 / 2}$ & 0.0097 & 0.0085 & 0.0034 & $201.760(5-37)$ & \\
\hline 203.728 & $3-32$ & $3 s^{2} 3 p^{3}{ }^{2} D_{5 / 2}-3 s^{2} 3 p^{2} 3 d^{2} D_{5 / 2}$ & 0.0867 & 0.0711 & 0.0231 & & bl Fe XIII \\
\hline 211.732 & $2-28$ & $3 s^{2} 3 p^{3}{ }^{2} \mathrm{D}_{3 / 2}-3 \mathrm{~s} 3 \mathrm{p}^{4}{ }^{2} \mathrm{P}_{1 / 2}$ & 0.0242 & 0.0203 & 0.0074 & $211.700(40-179)$ & \\
\hline 217.276 & $2-26$ & $3 \mathrm{~s}^{2} 3 \mathrm{p}^{3}{ }^{2} \mathrm{D}_{3 / 2}-3 \mathrm{~s} 3 \mathrm{p}^{4}{ }^{2} \mathrm{P}_{3 / 2}$ & 0.0345 & 0.0289 & 0.0100 & & \\
\hline 219.437 & $3-26$ & $3 \mathrm{~s}^{2} 3 \mathrm{p}^{3}{ }^{2} \mathrm{D}_{5 / 2}-3 \mathrm{~s} 3 \mathrm{p}^{4}{ }^{2} \mathrm{P}_{3 / 2}$ & 0.0864 & 0.0724 & 0.0251 & & \\
\hline 220.870 & $1-22$ & $3 s^{2} 3 p^{3}{ }^{4} S_{3 / 2}-3 s^{2} 3 p^{2} 3 d^{4} D_{5 / 2}$ & 0.0321 & 0.0273 & 0.0102 & & w \\
\hline 222.306 & $1-18$ & $3 s^{2} 3 p^{3}{ }^{4} S_{3 / 2}-3 s^{2} 3 p^{2} 3 d^{2} F_{5 / 2}$ & 0.0384 & 0.0334 & 0.0133 & & $\mathrm{w}$ \\
\hline 223.000 & $3-24$ & $3 s^{2} 3 p^{3}{ }^{2} D_{5 / 2}-3 s^{2} 3 p^{2} 3 d^{2} G_{7 / 2}$ & 0.0176 & 0.0156 & 0.0063 & & W \\
\hline 249.388 & $3-20$ & $3 s^{2} 3 p^{3}{ }^{2} D_{5 / 2}-3 s^{2} 3 p^{2} 3 d^{4} D_{7 / 2}$ & 0.0739 & 0.0635 & 0.0242 & $249.384(3-21)$ & \\
\hline 256.410 & $3-16$ & $3 s^{2} 3 p^{3}{ }^{2} D_{5 / 2}-3 s^{2} 3 p^{2} 3 d^{4} F_{7 / 2}$ & 0.0662 & 0.0572 & 0.0219 & & $\mathrm{bl}$ \\
\hline 283.443 & $2-12$ & $3 s^{2} 3 p^{3}{ }^{2} D_{3 / 2}-3 s^{2} 3 p^{2} 3 d^{2} P_{1 / 2}$ & 0.0166 & 0.0151 & 0.0070 & & \\
\hline 291.010 & $3-11$ & $3 s^{2} 3 p^{3}{ }^{2} D_{5 / 2}-3 s^{2} 3 p^{2} 3 d^{2} P_{3 / 2}$ & 0.0781 & 0.0681 & 0.0271 & $290.962(32-104)$ & \\
\hline 303.135 & $5-13$ & $3 s^{2} 3 p^{3}{ }^{2} \mathrm{P}_{3 / 2}-3 \mathrm{~s} 3 \mathrm{p}^{4}{ }^{2} \mathrm{~S}_{1 / 2}$ & 0.0096 & 0.0088 & 0.0041 & & bl \\
\hline 335.380 & $2-9$ & $3 s^{2} 3 p^{3}{ }^{2} D_{3 / 2}-3 s 3 p^{4}{ }^{2} D_{3 / 2}$ & 0.0458 & 0.0429 & 0.0214 & & $\begin{array}{l}\text { bl Fe XVI } \\
\text { bl Mg VIII }\end{array}$ \\
\hline 338.263 & $3-10$ & $3 s^{2} 3 p^{3}{ }^{2} D_{5 / 2}-3 s 3 p^{4}{ }^{2} D_{5 / 2}$ & 0.0897 & 0.0802 & 0.0350 & & \\
\hline 346.852 & $1-8$ & $3 s^{2} 3 p^{3}{ }^{4} S_{3 / 2}-3 s 3 p^{4}{ }^{4} P_{1 / 2}$ & 0.1085 & 0.0930 & 0.0375 & & \\
\hline 352.106 & $1-7$ & $3 s^{2} 3 p^{3}{ }^{4} S_{3 / 2}-3 s 3 p^{4}{ }^{4} P_{3 / 2}$ & 0.2193 & 0.1893 & 0.0778 & & \\
\hline 364.467 & $1-6$ & $3 s^{2} 3 p^{3}{ }^{4} S_{3 / 2}-3 s 3 p^{4}{ }^{4} P_{5 / 2}$ & 0.3865 & 0.3327 & 0.1345 & & \\
\hline 1242.01 & $1-5$ & $3 s^{2} 3 p^{3}{ }^{4} S_{3 / 2}-3 s^{2} 3 p^{3}{ }^{2} P_{3 / 2}$ & 0.1287 & 0.1283 & 0.0855 & & \\
\hline 1349.40 & $1-4$ & $3 s^{2} 3 p^{3}{ }^{4} S_{3 / 2}-3 s^{2} 3 p^{3}{ }^{2} P_{1 / 2}$ & 0.0709 & 0.0721 & 0.0508 & & \\
\hline 2169.76 & $1-3$ & $3 \mathrm{~s}^{2} 3 \mathrm{p}^{3}{ }^{4} \mathrm{~S}_{3 / 2}-3 \mathrm{~s}^{2} 3 \mathrm{p}^{3}{ }^{2} \mathrm{D}_{5 / 2}$ & 0.0782 & 0.0687 & 0.0310 & & \\
\hline 2406.41 & $1-2$ & $3 \mathrm{~s}^{2} 3 \mathrm{p}^{3} \mathrm{~S}_{3 / 2}-3 \mathrm{~s}^{2} 3 \mathrm{p}^{3}{ }^{2} \mathrm{D}_{3 / 2}$ & 0.4086 & 0.3880 & 0.2286 & & \\
\hline 2566.77 & $2-5$ & $3 s^{2} 3 p^{3}{ }^{2} D_{3 / 2}-3 s^{2} 3 p^{3}{ }^{2} P_{3 / 2}$ & 0.0772 & 0.0770 & 0.0513 & & \\
\hline 22063.0 & $2-3$ & $3 s^{2} 3 p^{3}{ }^{2} D_{3 / 2}-3 s^{2} 3 p^{3}{ }^{2} D_{5 / 2}$ & 0.0398 & 0.0350 & 0.0158 & & \\
\hline
\end{tabular}


J. Dudík et al.: Signatures of the non-Maxwellian $\kappa$-distributions in optically thin line spectra

Table B.5. Same as Table B.1, but for Fe XIII.

\begin{tabular}{|c|c|c|c|c|c|c|c|}
\hline$\lambda[\AA]$ & Levels & Level description & $I(\mathrm{Maxw})$ & $I(\kappa=5)$ & $I(\kappa=2)$ & Selfblends & Notes \\
\hline 196.525 & $4-26$ & $3 s^{2} 3 p^{2}{ }^{1} D_{2}-3 s^{2} 3 p 3 d^{1} F_{3}$ & 0.0564 & 0.0479 & 0.0133 & & \\
\hline 200.021 & $2-24$ & $3 s^{2} 3 p^{2}{ }^{3} P_{1}-3 s^{2} 3 p 3 d^{3} D_{2}$ & 0.1391 & 0.1142 & 0.0283 & & \\
\hline 201.126 & $2-23$ & $3 s^{2} 3 p^{2}{ }^{3} P_{1}-3 s^{2} 3 p 3 d^{3} D_{1}$ & 0.2363 & 0.1885 & 0.0425 & & bl Fe XII \\
\hline 202.044 & $1-20$ & $3 s^{2} 3 p^{2}{ }^{3} P_{0}-3 s^{2} 3 p 3 d^{3} P_{1}$ & 0.6461 & 0.4987 & 0.0996 & & \\
\hline 203.165 & $2-22$ & $3 s^{2} 3 p^{2}{ }^{3} P_{1}-3 s^{2} 3 p 3 d^{3} P_{0}$ & 0.0707 & 0.0579 & 0.0143 & & \\
\hline 203.826 & $3-25$ & $3 s^{2} 3 p^{2}{ }^{3} P_{2}-3 s^{2} 3 p 3 d^{3} D_{3}$ & 0.6762 & 0.5533 & 0.1361 & $\begin{array}{l}203.772(7-60) \\
203.795(3-24) \\
203.835(8-60)\end{array}$ & bl Fe XII \\
\hline 204.262 & $2-21$ & $3 s^{2} 3 p^{2}{ }^{3} P_{1}-3 s^{2} 3 p 3 d^{1} D_{2}$ & 0.0586 & 0.0485 & 0.0124 & & \\
\hline 204.942 & $3-23$ & $3 s^{2} 3 p^{2}{ }^{3} P_{2}-3 s^{2} 3 p 3 d^{3} D_{1}$ & 0.0700 & 0.0558 & 0.0126 & & \\
\hline 208.667 & $5-27$ & $3 \mathrm{~s}^{2} 3 \mathrm{p}^{2}{ }^{1} S_{0}-3 \mathrm{~s}^{2} 3 \mathrm{p} 3 \mathrm{~d}^{1} \mathrm{P}_{1}$ & 0.0228 & 0.0185 & 0.0044 & $\begin{array}{l}208.716(11-67) \\
208.754(9-57)\end{array}$ & \\
\hline 209.619 & $2-19$ & $3 s^{2} 3 p^{2}{ }^{3} P_{1}-3 s^{2} 3 p 3 d^{3} P_{2}$ & 0.0970 & 0.0801 & 0.0203 & $209.654(6-43)$ & \\
\hline 209.916 & $3-20$ & $3 s^{2} 3 p^{2}{ }^{3} P_{2}-3 s^{2} 3 p 3 d^{3} P_{1}$ & 0.1159 & 0.0895 & 0.0179 & & \\
\hline 213.768 & $3-19$ & $3 s^{2} 3 p^{2}{ }^{3} P_{2}-3 s^{2} 3 p 3 d^{3} P_{2}$ & 0.0958 & 0.0792 & 0.0201 & & \\
\hline 216.835 & $4-24$ & $3 s^{2} 3 p^{2}{ }^{1} D_{2}-3 s^{2} 3 p 3 d^{3} D_{2}$ & 0.0457 & 0.0375 & 0.0093 & $216.870(4-25)$ & \\
\hline 221.828 & $4-21$ & $3 s^{2} 3 p^{2}{ }^{1} D_{2}-3 s^{2} 3 p 3 d^{1} D_{2}$ & 0.1217 & 0.1006 & 0.0256 & $221.824(6-20)$ & \\
\hline 228.160 & $4-19$ & $3 s^{2} 3 p^{2}{ }^{1} D_{2}-3 s^{2} 3 p 3 d^{3} P_{2}$ & 0.0836 & 0.0691 & 0.0176 & & \\
\hline 239.030 & $3-16$ & $3 s^{2} 3 p^{2}{ }^{3} P_{2}-3 s^{2} 3 p 3 d^{3} F_{3}$ & 0.0785 & 0.0658 & 0.0173 & & \\
\hline 240.696 & $1-14$ & $3 s^{2} 3 p^{2}{ }^{3} P_{0}-3 s 3 p^{3}{ }^{3} S_{1}$ & 0.0744 & 0.0609 & 0.0151 & & \\
\hline 246.209 & $2-14$ & $3 \mathrm{~s}^{2} 3 \mathrm{p}^{2}{ }^{3} \mathrm{P}_{1}-3 \mathrm{~s} 3 \mathrm{p}^{3}{ }^{3} S_{1}$ & 0.1856 & 0.1519 & 0.0376 & $246.241(16-76)$ & bl Si VI \\
\hline 251.952 & $3-14$ & $3 \mathrm{~s}^{2} 3 \mathrm{p}^{2}{ }^{3} \mathrm{P}_{2}-3 \mathrm{~s} 3 \mathrm{p}^{3}{ }^{3} S_{1}$ & 0.3557 & 0.2912 & 0.0721 & & \\
\hline 256.400 & $4-17$ & $3 s^{2} 3 p^{2}{ }^{1} D_{2}-3 s 3 p^{3}{ }^{1} P_{1}$ & 0.0694 & 0.0581 & 0.0155 & & $\mathrm{bl}$ \\
\hline 261.743 & $4-15$ & $3 s^{2} 3 p^{2}{ }^{1} D_{2}-3 s^{2} 3 p 3 d^{3} F_{2}$ & 0.0393 & 0.0325 & 0.0083 & & \\
\hline 303.364 & $1-11$ & $\mathrm{~s}^{2} 3 \mathrm{p}^{2}{ }^{3} \mathrm{P}_{0}-3 \mathrm{~s} 3 \mathrm{p}^{3}{ }^{3} \mathrm{P}_{1}$ & 0.0624 & 0.0521 & 0.0140 & & bl \\
\hline 312.174 & $2-11$ & $3 \mathrm{~s}^{2} 3 \mathrm{p}^{2}{ }^{3} \mathrm{P}_{1}-3 \mathrm{~s} 3 \mathrm{p}^{3}{ }^{3} \mathrm{P}_{1}$ & 0.0912 & 0.0761 & 0.0205 & & \\
\hline 312.868 & $2-10$ & $3 s^{2} 3 p^{2}{ }^{3} P_{1}-3 s 3 p^{3}{ }^{3} P_{0}$ & 0.0446 & 0.0386 & 0.0117 & & \\
\hline 318.130 & $4-13$ & $3 \mathrm{~s}^{2} 3 \mathrm{p}^{2}{ }^{1} \mathrm{D}_{2}-3 \mathrm{~s} 3 \mathrm{p}^{3}{ }^{1} \mathrm{D}_{2}$ & 0.0621 & 0.0561 & 0.0188 & & $\mathrm{bl}$ ? \\
\hline 320.800 & $3-12$ & $3 s^{2} 3 p^{2}{ }^{3} P_{2}-3 s 3 p^{3}{ }^{3} P_{2}$ & 0.1566 & 0.1361 & 0.0415 & & \\
\hline 348.183 & $1-7$ & $\mathrm{~s}^{2} 3 \mathrm{p}^{2}{ }^{3} \mathrm{P}_{0}-3 \mathrm{~s} 3 \mathrm{p}^{3}{ }^{3} \mathrm{D}_{1}$ & 0.2185 & 0.1793 & 0.0465 & & \\
\hline 359.644 & $2-8$ & $3 s^{2} 3 p^{2}{ }^{3} P_{1}-3 s 3 p^{3}{ }^{3} D_{2}$ & 0.2180 & 0.1886 & 0.0580 & & \\
\hline 368.164 & $3-9$ & $3 s^{2} 3 p^{2}{ }^{3} P_{2}-3 s 3 p^{3}{ }^{3} D_{3}$ & 0.2145 & 0.1877 & 0.0595 & & bl Mg IX \\
\hline 487.042 & $2-6$ & $3 \mathrm{~s}^{2} 3 \mathrm{p}^{2}{ }^{3} \mathrm{P}_{1}-3 \mathrm{~s} 3 \mathrm{p}^{3}{ }^{5} S_{2}$ & 0.0286 & 0.0274 & 0.0115 & & \\
\hline 510.042 & $3-6$ & $3 \mathrm{~s}^{2} 3 \mathrm{p}^{2}{ }^{3} \mathrm{P}_{2}-3 \mathrm{~s} 3 \mathrm{p}^{3}{ }^{5} S_{2}$ & 0.0486 & 0.0466 & 0.0195 & & \\
\hline 2579.54 & $2-4$ & $3 \mathrm{~s}^{2} 3 \mathrm{p}^{2}{ }^{3} \mathrm{P}_{1}-3 \mathrm{~s}^{2} 3 \mathrm{p}^{2}{ }^{1} \mathrm{D}_{2}$ & 0.2933 & 0.2719 & 0.1157 & & \\
\hline 3388.91 & $3-4$ & $3 s^{2} 3 p^{2}{ }^{3} P_{2}-3 s^{2} 3 p^{2}{ }^{1} D_{2}$ & 0.3585 & 0.3323 & 0.1414 & & \\
\hline 10749.0 & $1-2$ & $3 s^{2} 3 p^{2}{ }^{3} \mathrm{P}_{0}-3 s^{2} 3 p^{2}{ }^{3} \mathrm{P}_{1}$ & 1.0000 & 0.8658 & 0.2871 & & \\
\hline 10801.0 & $2-3$ & $3 s^{2} 3 p^{2}{ }^{3} \mathrm{P}_{1}-3 s^{2} 3 p^{2}{ }^{3} \mathrm{P}_{2}$ & 0.7816 & 0.6789 & 0.2282 & & \\
\hline
\end{tabular}

